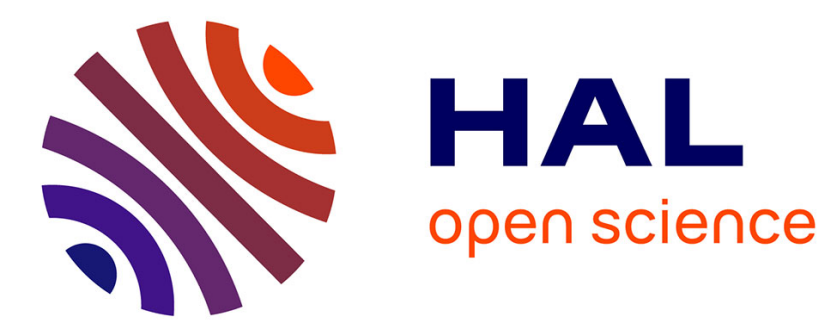

\title{
Apparent Flexoelectricity due to Heterogeneous Piezoelectricity
}

Julien Yvonnet, X Chen, P Sharma

\section{To cite this version:}

Julien Yvonnet, X Chen, P Sharma. Apparent Flexoelectricity due to Heterogeneous Piezoelectricity. Journal of Applied Mechanics, 2020, 87 (11), pp.111003. hal-03037302

\section{HAL Id: hal-03037302 https://hal.science/hal-03037302}

Submitted on 3 Dec 2020

HAL is a multi-disciplinary open access archive for the deposit and dissemination of scientific research documents, whether they are published or not. The documents may come from teaching and research institutions in France or abroad, or from public or private research centers.
L'archive ouverte pluridisciplinaire $\mathbf{H A L}$, est destinée au dépôt et à la diffusion de documents scientifiques de niveau recherche, publiés ou non, émanant des établissements d'enseignement et de recherche français ou étrangers, des laboratoires publics ou privés. 


\title{
Apparent Flexoelectricity due to Heterogeneous Piezoelectricity
}

\author{
J. Yvonnet ${ }^{a, *}, X$. Chen ${ }^{a, b}$, P. Sharma ${ }^{c}$ \\ ${ }^{a}$ Univ Gustave Eiffel, CNRS, MSME UMR 8208, F-77454 Marne-la-Vallée, \\ France \\ ${ }^{\mathrm{b}}$ Key Laboratory of Traffic Safety on Track, Ministry of Education, School of \\ Traffic 85 Transportation Engineering, Central South University, Changsha 410075, \\ China \\ ${ }^{\mathrm{c}}$ Department of Mechanical Engineering, Department of Physics, University of \\ Houston, Houston, TX, 77204
}

\begin{abstract}
Recent work has highlighted how the phenomenon of flexoelectricity can masquerade as piezoelectricity. This notion can not only be exploited to create artificial piezoelectric-like materials without using piezoelectric materials, but may also explain measurement artifacts in dielectrics. In this article, we show that the reverse is also possible and potentially advantageous in certain situations (such as energy harvesting). By constructing a computational homogenization approach predicated on the finite element method, we argue that composites made of piezoelectric phases can conspire to endow the material with a distinct overall flexoelectric-like response even though the native flexoelectricity of the constituent materials is negligible. Full finite element procedures for numerical evaluation of the different effective tensors, including the flexoelectric tensor, are provided. Numerical investigations are conducted, showing variation of the effective flexoelectric properties with respect to local geometry and properties of the composite in piezoelectric-piezoelectric and polymer-piezoelectric composites. We find that the flexoelectric response can be tuned to nearly five times higher than the constituents.
\end{abstract}

Key words: Computational homogenization, Strain gradient, Second-order homogenization, Finite Element, flexoelectricity

\footnotetext{
* Correspondance to J. Yvonnet

Email address: julien.yvonnet@univ-paris-est.fr (J. Yvonnet ).
} 


\section{Introduction}

The ability of certain materials that can convert mechanical deformation into electricity and vice-versa is a prized property with applications that are well recorded in the vast literature on so-called "smart materials" $[1,2,3,4,5,6,7$, $8,9,10]$. Piezoelectrics are the traditional material system that embody this concept of electromechanical coupling and have found applications in energy harvesting [11, 12], artificial muscles [13], sensors [14], actuators [15], robotics among many others [16].

More recently, significant attention has been paid to another electromechanical mechanism-flexoelectricity-which links strain gradients to electric fields ${ }^{1}$ and electric field gradients to mechanical deformation $[18,19,20,21,22,23$, $24,25,26,27,28,29,30]$. This is in contrast to piezoelectrics which exhibit a linear coupling between uniform strain and uniform electric fields. The following comparison between the two phenomena is important to understand the motivation underpinning our work:

(1) The advantage of flexoelectricity over piezoeletricity is that it is universal and all dielectrics exhibit this phenomenon [27, 25]. This is in sharp contrast to piezoelectric materials that must possess a non-centrosymmetric crystal structure and is thus is limited in nature to materials such as quartz or ferroelectrics like barium titanate, lead zirconate titanate among others.

(2) The price of the universality of flexoelectricity is that the electromechanical coupling is rather weak. The intrinsic value of flexoelectric properties for most dielectrics is such that unless extremely large strain gradients are present (usually only possible for nanostructures or at the nanoscale), the induced electric fields are small $[25,31]$.

(3) One of the most important applications of piezoelectricity is the prospects of energy harvesting from mechanical motion [20, 26, 25, 32]. Flexure motion, in particular, is the most facile manner in which mechanical deformation can be harvested into electrical energy. However, that said, simple bending of piezoelectrics is not very efficient to generate electricity since the compression on one side of the neutral axis produces electrical po-

\footnotetext{
${ }^{1}$ Or alternatively to polarization gradients. Electrostatics offers a choice of multiple independent variables and theories of electromechanical coupling can superficially appear different based on this choice. We refer to the exposition by Liu [17] which outlines this and other related aspects in detail
} 
larization that tends to counteract the polarization in the tensile portion of the flexing element. A simple recourse is to use bimorphs-i.e. placement of an inactive element that shift the neutral axis. Flexoelectricity, if it were to be substantive, does not suffer from this handicap and is perfectly suited to directly convert flexure motion into usable electricity $[33,34,35]$.

In hard ceramics such as $\mathrm{BaTi}_{3}$, the flexoelectric coefficients can be relatively high [27], but the required local high strain gradient may be limited by the high stiffness and brittle nature of these materials. To our knowledge, there are very few such exceptional materials like $\mathrm{BaTiO}_{3}{ }^{2}$. Two possible ways to increase flexoelectricity is to consider electrets, i.e. to insert fixed charges in the material $[36,35]$, or increase the local strain gradient by considering nanostructures. In [35], Rahmati et al. [35] analyzed the behavior of electrets under nonlinear bending and showed significant enhancement of flexoelectricity and this notion was experimentally verified in [36].

Earlier works focused on exploiting flexoelectricity to design piezoelectric-like materials without using piezoelectrics [37, 38]. A related notion is also of how flexoelectricity could mimic piezoelectricity [39]. In this work we examine the reverse problem. Is it possible to use piezoelectrics to create a very large flexoelectric response? As motivated earlier, if the flexoelectric response is engineered to be substantive, energy harvesting can be made more efficient especially for small scale structures. Accordingly in this work we consider the use of architectured materials composed of heterogeneous piezoelectric phases with an aim of achieving a large emergent flexoelectric response - one that may effectively overshadow the materials native flexoelectricity. Indeed, it has been shown in [40] that the recent progresses of material engineering and 3D printing techniques allows designing heterogeneous piezoelectric structures or materials with "on-demand" microstructures.

It is germane here to mention several relevant papers. Guinovart-Sanjuan et al. [41] used a two scale asymptotic homogenization method to derive the homogenized flexoelectric behavior in 1D rods. In [42], the effective flexoelectric and piezoelectric behavior of fiber-reinforced nanocomposites with local flexoelectric fibers was derived using an analytical approach. In [43], a homogenization technique was developed to predict the apparent piezo and flexoelectric properties for specific (pantographic) structures with flexoelectric pivots and bars. In [44] Mohammadi et al. considered a heterogeneous membrane

$\overline{2}$ Flexoelectricity in soft materials is also an actively studied subject [34, 35]. The value of coupling is, like most hard dielectrics, also small although higher strain gradients may be easily achieved 
and derived its homogenized flexoelectric behavior. In [45], the effective flexoelectricity was studied in inhomogeneous dielectrics using direct (local) calculations on microstructures. As already indicated, emergent flexoelectricity has also been explored with embedded charges in materials - both theoretically and experimentally $[35,36]$. Another promising approach is the use of topology optimization to design heterogeneous piezoelectric structures so as to maximize the flexoelectric effects. In [46], Nanthakumar et al. developed a topology optimization framework for heterogeneous piezoelectric structures and demonstrated a significant enhancement in energy conversion. In [47], the same authors extended this framework to multi materials.

In all mentioned works, either analytical homogenization methods or direct numerical calculations on structures or microstructures were performed to analyze their apparent properties [48]. Numerical formulations, i.e. finite element formulations, can be found e.g. in $[49,50,51]$ and more general approaches that tackle computational electrostatics may be found in the following and citations therein $[52,53]$. While numerical approaches using e.g. FEM of FFT for homogenization of piezoelectric composites in absence of strain gradient is well-known (see e.g.[54, 55]), a computational homogenization framework for effective flexoelectric materials is so far not available. Such homogenization framework is required to study and optimize the above-mentioned related applications without the need to solve the full heterogeneous structure, especially when the ratio between the characteristic dimensions of the heterogeneities and those of the structure is very small (separated scales).

In the present paper we achieve our goal of designing flexoelectricity by using heterogeneous piezoeletric phases through the development of a computational homogenization framework. Specifically, the effective behavior is defined as a Mindlin strain gradient medium $[56,57]$ enriched with energetic terms associated with the electro mechanical coupling corresponding to flexoelectricity. A finite element procedure is described to compute the different homogenized (effective) tensors on a Representative Volume Element (RVE), including the full fourth-order flexoelectric tensor.

This paper is organized as follows. In section 2, the notations used in this work are summarized. In section 3, the equations of the local piezoelectric problem on the RVE are presented. The homogenized model and the method to compute the effective tensors is presented in section 4. Finally, numerical investigations are performed in section 5 to analyze the effective flexoelectric behavior of piezoelectric composites, including polymer-piezoelectric and piezoelectric-piezoelectric microstructures. 


\section{Preliminary notations}

Vectors and second-order tensors, as well as matrices, are denoted by bold letters A. Third-order tensors are denoted by calligraphic uppercase letters $\mathcal{G}$, fourth-order, fifth-order and sixth-order tensors are denoted by double case letters $\mathbb{A}$. Double contraction of indices for second order tensors $\mathbf{A}$ and $\mathbf{B}$ is denoted by $\mathbf{A}: \mathbf{B}=A_{i j} B_{i j}$, dot product for two vectors $\mathbf{a}$ and $\mathbf{b}$ by $\mathbf{a} \cdot \mathbf{b}=a_{i} b_{i}$, and simple contraction of indices for a second order tensor $\mathbf{A}$ and a vector $\mathbf{b}$ is denoted by $(\mathbf{A} \cdot \mathbf{b})_{i}=A_{i j} b_{j}$. For the purpose of this paper, we introduce the triple contraction of indices for two third-order tensors $\mathcal{G}$ and $\mathcal{B}$ as: $\mathcal{G} \vdots \mathcal{B}=G_{i j k} B_{i j k}$. The gradient operator is denoted by $\nabla($.$) and the$ divergence operator by $\nabla \cdot($.$) .$

Let $\mathbf{u}$ the displacement vector and $\mathbf{x}$ a material coordinate, we define:

$$
\varepsilon_{i j}=\frac{1}{2}\left(\frac{\partial u_{i}}{\partial x_{j}}+\frac{\partial u_{j}}{\partial x_{i}}\right)
$$

and the third-order strain gradient tensor as:

$$
(\nabla \varepsilon)_{i j k}=\frac{1}{2}\left(\frac{\partial^{2} u_{i}}{\partial x_{j} \partial x_{k}}+\frac{\partial^{2} u_{j}}{\partial x_{i} \partial x_{k}}\right) .
$$

The second gradient displacements (third-order) tensor is defined as

$$
\mathcal{G}_{i j k}=\frac{\partial^{2} u_{i}}{\partial x_{j} \partial x_{k}}
$$

It can be shown (see $[57,58]$ that these two tensors are related by

$$
\mathcal{G}_{i j k}=(\nabla \varepsilon)_{i j k}+(\nabla \varepsilon)_{i k j}-(\nabla \varepsilon)_{j k i} .
$$

Let $\phi$ be the electric potential, we define the electric field as:

$$
E_{i}=-\frac{\partial \phi}{\partial x_{i}}
$$

\section{Micro RVE problem}

We assume a periodic medium characterized by an RVE defined in a domain $\Omega \subset \mathbb{R}^{2}$ whose boundary is denoted by $\partial \Omega$ (see Fig. 1 (c)). The RVE is assumed to be subjected to a homogeneous strain $\bar{\varepsilon}$, a homogeneous strain gradient $\overline{\nabla \varepsilon}$ and a homogeneous electric field $\overline{\mathbf{E}}$. The RVE is characterized by 


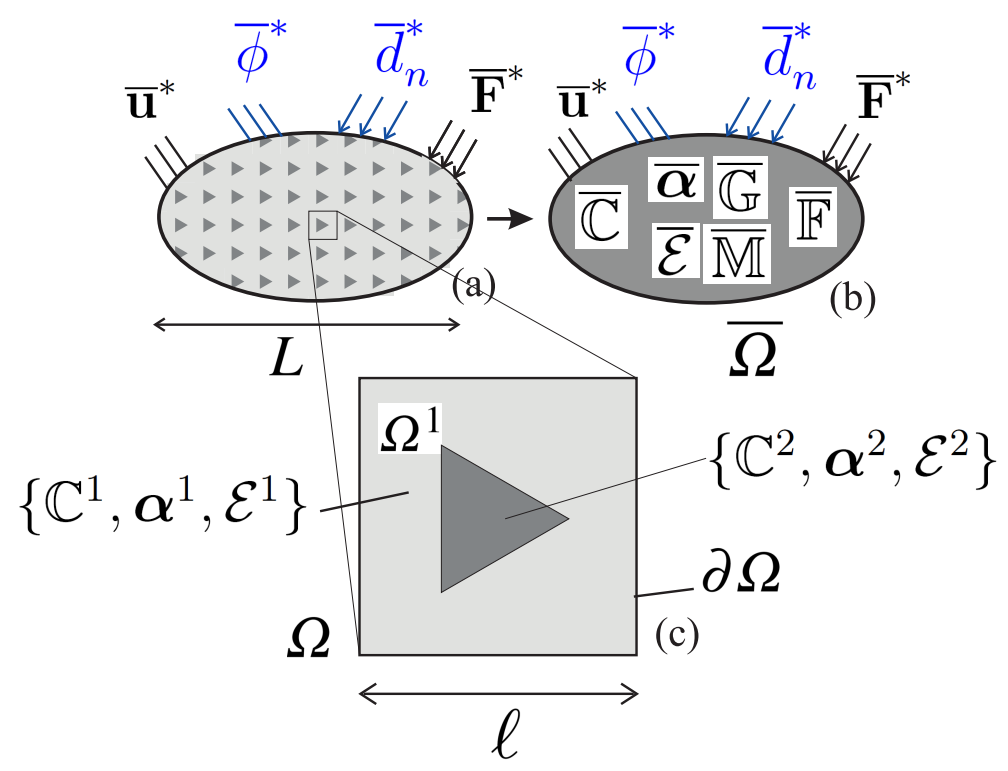

Fig. 1. (a) heterogeneous structure; (b) Equivalent piezo-flexoelectric homogeneous structure; (c) RVE embedding local piezoelectric phases.

a size $\ell$. Within the RVE, the different phases are assumed to be locally linear piezoelectric and characterized by fourth-order elastic tensors $\mathbb{C}^{i}$, second-order tensors of dielectric properties $\boldsymbol{\alpha}^{i}$ and third order tensors of piezoelectric properties $\mathcal{E}^{i}, i=1,2, \ldots, N$, with $N$ the number of phases. The local equations are defined as:

$$
\begin{aligned}
& \nabla \cdot \boldsymbol{\sigma}(\mathbf{x})=0 \text { in } \Omega, \\
& \nabla \cdot \mathbf{d}=0 \text { in } \Omega,
\end{aligned}
$$

where $\mathbf{d}$ denotes the dielectric displacement, and $\boldsymbol{\sigma}$ is the Cauchy stress tensor with:

$$
\sigma_{i j}(\mathbf{x})=\mathbb{C}_{i j k l}(\mathbf{x}) \varepsilon_{k l}(\mathbf{x})-\mathcal{E}_{k i j}(\mathbf{x}) E_{k}(\mathbf{x})
$$

and

$$
d_{i}(\mathbf{x})=\mathcal{E}_{i j k}(\mathbf{x}) \varepsilon_{j k}(\mathbf{x})+\alpha_{i j}(\mathbf{x}) E_{j}(\mathbf{x})
$$

Quadratic boundary conditions have been introduced in several works (see e.g. $[59,60,61])$ to prescribe an effective strain gradient over the RVE:

$$
\mathbf{u}(\mathbf{x})=\overline{\boldsymbol{\varepsilon}} \cdot \mathbf{x}+\frac{1}{2} \overline{\mathcal{G}}: \mathbf{x} \otimes \mathbf{x}+\tilde{\mathbf{u}}(\mathbf{x}) \text { on } \partial \Omega,
$$

where $\overline{\mathcal{G}}$ depends on $\overline{\nabla \varepsilon}$ according to (4) and $\tilde{\mathbf{u}}(\mathbf{x})$ is either zero or periodic on $\partial \Omega$. We have shown in $[58,62]$ that such boundary conditions alone can induce several issues such as spurious gradient terms and a non-convergence of the 
effective higher order effective coefficients with respect to the RVE size. In [58], we have introduced body forces in addition to the above quadratic boundary conditions as an ingredient to remove the above mentioned spurious effects. The body forces are such that when the RVE is homogeneous, the local strain field is strictly linear. In this work, we extend this idea to the coupled electromechanical problem. Then, for a linear strain field $\overline{\nabla \varepsilon} \cdot \mathbf{x}$ in the RVE with constant local properties $\mathbb{C}^{0}$ and $\mathcal{E}^{0}$, Eqs. (6) and (7) together with (8) and (9) lead to

$$
\begin{aligned}
& \sigma_{i j, j}=C_{i j k l}^{0}(\overline{\nabla \varepsilon})_{k l j}, \\
& d_{i, i}=\mathcal{E}_{p j k}^{0}(\overline{\nabla \varepsilon})_{j k p} .
\end{aligned}
$$

Choosing $\mathbb{C}^{0}=\overline{\mathbb{C}}$ and $\mathcal{E}^{0}=\overline{\mathcal{E}}$ as the effective elastic and piezoelectric tensor guarantees that when the material is homogeneous, the local properties go to constant values equal to the local material properties. The definition of $\overline{\mathbb{C}}$ and $\overline{\mathcal{E}}$ is provided in section 4.2 . Then, an alternative local problem is defined as:

$$
\begin{aligned}
& \nabla \cdot \boldsymbol{\sigma}(\mathbf{u}(\mathbf{x}))=\mathbf{f}(\overline{\nabla \boldsymbol{\varepsilon}}) \forall \mathbf{x} \in \Omega, \\
& \nabla \cdot \mathbf{d}(\mathbf{x})=r(\overline{\nabla \varepsilon}) \forall \mathbf{x} \in \Omega,
\end{aligned}
$$

where

$$
\begin{aligned}
& f_{i}=\overline{\mathcal{C}}_{i j k l}(\overline{\nabla \varepsilon})_{k l j} \\
& r=\overline{\mathcal{E}}_{i j k}(\overline{\nabla \varepsilon})_{j k i} .
\end{aligned}
$$

The effective electric field can be prescribed using boundary conditions in the form (see e.g. [63]):

$$
\phi(\mathbf{x})=-\overline{\mathbf{E}} \cdot \mathbf{x}+\tilde{\phi}(\mathbf{x}) \text { on } \partial \Omega
$$

where $\tilde{\phi}(\mathbf{x})$ is either zero or a periodic fluctuation over $\Omega$. To summarize, we define the localization problem to be solved on the RVE as follows: 
Given $\bar{\varepsilon}, \overline{\nabla \varepsilon}$ and $\overline{\mathbf{E}}$, find $\boldsymbol{\varepsilon}(\mathbf{x})$ and $\phi(\mathbf{x})$ such that:

$$
\nabla \cdot \boldsymbol{\sigma}(\mathbf{u}(\mathbf{x}))=\mathbf{f}(\overline{\nabla \boldsymbol{\varepsilon}}) \forall \mathbf{x} \in \Omega,
$$

where $\mathbf{f}$ is given by (15),

$$
\nabla \cdot \mathbf{d}(\mathbf{x})=r(\overline{\nabla \varepsilon}) \forall \mathbf{x} \in \Omega,
$$

where $r$ is given in (16),

$$
\begin{aligned}
& \boldsymbol{\sigma}(\mathbf{x})=\mathbb{C}(\mathbf{x}): \varepsilon(\mathbf{x})-\mathcal{E}^{T}(\mathbf{x}) \cdot \overline{\mathbf{E}}, \\
& \mathbf{d}(\mathbf{x})=\mathcal{E}(\mathbf{x}): \boldsymbol{\varepsilon}(\mathbf{x})+\boldsymbol{\alpha}(\mathbf{x}) \cdot \overline{\mathbf{E}}
\end{aligned}
$$

and subjected to:

$$
\begin{aligned}
& \mathbf{u}(\mathbf{x})=\frac{1}{2} \overline{\mathcal{G}}: \mathbf{x} \otimes \mathbf{x}+\overline{\boldsymbol{\varepsilon}} \cdot \mathbf{x}+\tilde{\mathbf{u}}(\mathbf{x}) \text { on } \partial \Omega \\
& \phi(\mathbf{x})=-\overline{\mathbb{E}} \cdot \mathbf{x}+\tilde{\phi}(\mathbf{x}) \text { on } \partial \Omega
\end{aligned}
$$

Problem (18)-(23) can be solved classically by FEM (see e.g. [63]). For the self-completeness of the paper, we have introduced the details in Appendix 8. In this paper, we restrict the boundary conditions to purely quadratic ones, i.e. $\tilde{\mathbf{u}}(\mathbf{x})=0, \tilde{\phi}(\mathbf{x})=0$. In [58] and [62], we have discussed the link between the above quadratic boundary conditions and asymptotic expansion homogenization techniques in the context of purely mechanical strain gradient problems. Even though extensions are required, the same ideas can be applied in the context of electromechanical coupling.

\section{Effective piezo-flexoelectric model}

In this section, the effective piezo-flexoelectric model is provided, and the definition of its different tensors is provided from the RVE micro problem solutions.

\subsection{Macroscopic model}

We introduce the energy density function (electrical enthalpy density) of an effective piezo-flexo electric material as composed to classical terms related to piezoelectric materials, terms of a Mindlin strain gradient model [56, 57], as well as a term coupling strain gradient and electric current (see e.g. [64]):

$$
\bar{W}=\frac{1}{2} \bar{\varepsilon}: \overline{\mathbb{C}}: \bar{\varepsilon}-\overline{\mathbf{E}} \cdot \overline{\mathcal{E}}: \bar{\varepsilon}-\frac{1}{2} \overline{\mathbf{E}} \cdot \overline{\boldsymbol{\alpha}} \cdot \overline{\mathbf{E}}
$$




$$
+\overline{\mathbf{E}} \cdot \overline{\mathbb{F}}: \overline{\nabla \varepsilon}+\bar{\varepsilon}: \overline{\mathbb{M}}: \overline{\nabla \varepsilon}+\frac{1}{2} \overline{\nabla \varepsilon}: \overline{\mathbb{G}}: \overline{\nabla \varepsilon}
$$

where $\overline{\mathbb{C}}$ denotes the fourth-order effective elastic tensor, $\overline{\boldsymbol{\alpha}}$ is the second-order effective dielectric tensor, $\overline{\mathcal{E}}$ is the third-order effective piezoelectric tensor, $\overline{\mathbb{F}}$ is a fourth-order flexoelectric tensor coupling electric field and strain gradient, $\overline{\mathbb{M}}$ is a fifth-order tensor coupling first and second order strains, and $\overline{\mathbb{G}}$ is the sixth-order strain gradient elasticity tensor. Note that in the above model, and in contrast to [64], we neglected the terms involving electric current gradient for the sake of simplicity. However, the present procedure could be extended to evaluating the terms associated to the electric field gradient in future studies.

Assuming only perfect interfaces between phases, the effective strain and electric fields are classically defined as:

$$
\bar{\varepsilon}=\langle\varepsilon(\mathbf{x})\rangle, \quad \overline{\mathbf{E}}=\langle\mathbf{E}(\mathbf{x})\rangle, \quad \overline{\nabla \varepsilon}=\langle\nabla \varepsilon(\mathbf{x})\rangle .
$$

It has been shown in several works (see e.g. $[65,66]$ ) that using quadratic boundary conditions as in section 3 , the relation $\overline{\nabla \varepsilon}=\langle\nabla \varepsilon(\mathbf{x})\rangle$ does not hold. Some attempts have been made to correct this point, such as in [66]. In the present work, we do not intend to satisfy this relationship exactly and only consider the definition of $\overline{\nabla \varepsilon}$ as an applied macroscopic quantity defined through the boundary conditions (22) and body forces in (18) and (19). This point could be improved in future studies.

Then the effective stress tensor $\overline{\boldsymbol{\sigma}}$, effective electric displacement $\overline{\mathbf{d}}$ and effective hyperstress tensor $\overline{\mathcal{S}}$ are defined, respectively, by:

$$
\begin{aligned}
& \overline{\mathbf{d}}=-\frac{\partial \bar{W}}{\partial \overline{\mathbf{E}}}=\overline{\mathcal{E}}: \overline{\boldsymbol{\varepsilon}}+\overline{\boldsymbol{\alpha}} \cdot \overline{\mathbf{E}}-\overline{\mathbb{F}}: \overline{\nabla \varepsilon} \\
& \overline{\boldsymbol{\sigma}}=\frac{\partial \bar{W}}{\partial \bar{\varepsilon}}=\overline{\mathbb{C}}: \bar{\varepsilon}-\overline{\mathcal{E}}^{T} \cdot \mathbf{E}+\overline{\mathbb{M}}: \overline{\nabla \varepsilon} \\
& \overline{\mathcal{S}}=\frac{\partial \bar{W}}{\partial \overline{\nabla \varepsilon}}=\overline{\mathbb{M}}^{T}: \bar{\varepsilon}+\overline{\mathbb{F}}^{T} \cdot \overline{\mathbf{E}}+\overline{\mathbb{G}}: \overline{\nabla \varepsilon}
\end{aligned}
$$

where $\left(\overline{\mathbb{F}}^{T} \cdot \overline{\mathbf{E}}\right)_{i}=\overline{\mathbb{F}}_{i j k l} \bar{E}_{i}$.

Let $\bar{\Omega} \subset \mathbb{R}^{2}$ a domain associated with the homogeneous strain gradient medium and $\partial \bar{\Omega}$ its boundary (see Fig. 1 (c)), balance equations are given by (see e.g. $[56])$ :

$$
\begin{aligned}
& \nabla \cdot \overline{\boldsymbol{\sigma}}-\nabla \cdot(\nabla \cdot \overline{\mathcal{S}})=0 \text { in } \bar{\Omega} \\
& \nabla \cdot \overline{\mathbf{d}}=0 \text { in } \Omega
\end{aligned}
$$


with boundary conditions:

$$
\begin{aligned}
& \overline{\mathbf{u}}=\overline{\mathbf{u}}^{*} \quad \text { on } \partial \bar{\Omega}_{u}, \\
& \overline{\boldsymbol{\sigma}} \cdot \overline{\mathbf{n}}-(\nabla \cdot \overline{\mathcal{S}}) \cdot \overline{\mathbf{n}}-\overline{\mathbf{F}}^{*}=0 \quad \text { on } \partial \bar{\Omega}_{F}, \\
& \overline{\mathcal{S}} \cdot \overline{\mathbf{n}} \otimes \overline{\mathbf{n}}=0 \quad \text { on } \partial \bar{\Omega}
\end{aligned}
$$

(see e.g. [17] for a justification), and where $\partial \bar{\Omega}_{u}$ and $\partial \bar{\Omega}_{F}$ are the Dirichlet and Neumann parts of the boundary $\partial \bar{\Omega}$, and:

$$
\begin{aligned}
& \bar{\phi}=\bar{\phi}^{*} \quad \text { on } \partial \bar{\Omega}_{\phi}, \\
& \overline{\mathbf{d}} \cdot \overline{\mathbf{n}}=\bar{d}_{n}^{*} \quad \text { on } \partial \bar{\Omega}_{d},
\end{aligned}
$$

where $\partial \bar{\Omega}_{\phi}$ and $\partial \bar{\Omega}_{d}$ are the Dirichlet and Neumann parts of the boundary $\partial \bar{\Omega}$ for the dielectric problem. This problem can be solved with appropriate $C^{1}$ finite elements discretizations (see e.g. [51, 67]).

\subsection{Definition of effective tensors}

The problem (18)-(23) being linear, using the superposition principle, the local strain field $\boldsymbol{\varepsilon}(\mathbf{x})$ and local electric fields $\mathbf{E}(\mathbf{x})$ can be expressed as:

$$
\begin{aligned}
& \varepsilon(\mathbf{x})=\mathbb{A}^{0}(\mathbf{x}): \bar{\varepsilon}+\mathcal{B}^{0}(\mathbf{x}) \cdot \overline{\mathbf{E}}+\mathbb{A}^{1}(\mathbf{x}): \overline{\nabla \varepsilon} \\
& \mathbf{E}(\mathbf{x})=\mathcal{D}^{0}(\mathbf{x}): \bar{\varepsilon}+\mathbf{h}^{0}(\mathbf{x}) \cdot \overline{\mathbf{E}}+\mathbb{D}^{1}(\mathbf{x}) \vdots \overline{\nabla \varepsilon}
\end{aligned}
$$

where:

- $\mathbb{A}_{i j k l}^{0}(\mathbf{x})$ is the strain solution $\varepsilon_{i j}(\mathbf{x})$ solution of (18)-(23) with $\overline{\boldsymbol{\varepsilon}}=\frac{1}{2}\left(\mathbf{e}_{k} \otimes \mathbf{e}_{l}+\mathbf{e}_{l} \otimes \mathbf{e}_{k}\right)$, $\overline{\mathbf{E}}=\mathbf{0}, \overline{\nabla \varepsilon}=\mathbf{0}$.

- $\mathcal{B}_{i j k}^{0}(\mathbf{x})$ is the strain solution $\varepsilon_{i j}(\mathbf{x})$ solution of (18)-(23) with $\overline{\boldsymbol{\varepsilon}}=\mathbf{0}, \overline{\mathbf{E}}=\mathbf{e}_{k}$, $\overline{\nabla \varepsilon}=\mathbf{0}$.

- $\mathbb{A}_{i j k l}^{1}(\mathbf{x})$ is the strain solution $\varepsilon_{i j}(\mathbf{x})$ solution of (18)-(23) with $\overline{\boldsymbol{\varepsilon}}=\mathbf{0}, \overline{\mathbf{E}}=\mathbf{0}$, $\overline{\nabla \varepsilon}=\frac{1}{2}\left(\mathbf{e}_{k} \otimes \mathbf{e}_{l}+\mathbf{e}_{l} \otimes \mathbf{e}_{k}\right) \otimes \mathbf{e}_{m}$.

- $\mathcal{D}_{i j k}^{0}(\mathbf{x})$ is the electric field solution $E_{i}(\mathbf{x})$ solution of (18)-(23) with $\bar{\varepsilon}=$ $\frac{1}{2}\left(\mathbf{e}_{j} \otimes \mathbf{e}_{k}+\mathbf{e}_{k} \otimes \mathbf{e}_{j}\right), \overline{\mathbf{E}}=\mathbf{0}, \overline{\nabla \varepsilon}=\mathbf{0}$.

- $h_{i j}^{0}(\mathbf{x})$ is the electric field solution $E_{i}(\mathbf{x})$ solution of (18)-(23) with $\overline{\boldsymbol{\varepsilon}}=\mathbf{0}$, $\overline{\mathbf{E}}=\mathbf{e}_{j}, \overline{\nabla \varepsilon}=\mathbf{0}$.

- $\mathcal{D}_{i j k l}^{1}(\mathbf{x})$ is the electric field solution $E_{i}(\mathbf{x})$ solution of (18)-(23) with $\overline{\boldsymbol{\varepsilon}}=\mathbf{0}$, $\overline{\mathbf{E}}=\mathbf{0}, \overline{\nabla \varepsilon}=\frac{1}{2}\left(\mathbf{e}_{j} \otimes \mathbf{e}_{k}+\mathbf{e}_{k} \otimes \mathbf{e}_{j}\right) \otimes \mathbf{e}_{l}$, 
where $\mathbf{e}_{i}$ are unitary basis vectors. In [58], we have introduced a correction on the localization operators associated with the strain gradient tensor to separate purely strain gradient effects and other loads, and which has been shown also as a second ingredient to remove spurious strain gradient remaining effects in a homogeneous medium. Following [58], we introduce a corrected expression of the localized fields as:

$$
\begin{aligned}
& \varepsilon(\mathbf{x})=\mathbb{A}^{0}(\mathbf{x}): \bar{\varepsilon}+\mathcal{B}^{0}(\mathbf{x}) \cdot \overline{\mathbf{E}}+\tilde{\mathbb{A}}^{1}(\mathbf{x}): \overline{\nabla \varepsilon} \\
& \mathbf{E}(\mathbf{x})=\mathcal{D}^{0}(\mathbf{x}): \overline{\boldsymbol{\varepsilon}}+\mathbf{h}^{0}(\mathbf{x}) \cdot \overline{\mathbf{E}}+\tilde{\mathbb{D}}^{1}(\mathbf{x}): \overline{\nabla \varepsilon}
\end{aligned}
$$

with

$$
\tilde{\mathbb{A}}^{1}(\mathbf{x})=\mathbb{A}^{1}(\mathbf{x})-\mathbb{A}^{0}(\mathbf{x}) \otimes \mathbf{x}
$$

and

$$
\tilde{\mathbb{D}}^{1}(\mathbf{x})=\mathbb{D}^{1}(\mathbf{x})-\mathcal{D}^{0}(\mathbf{x}) \otimes \mathbf{x}
$$

Computing the effective energy of the system:

$$
\begin{aligned}
& \bar{W}=\frac{1}{2}\langle\varepsilon(\mathbf{x}): \mathbb{C}(\mathbf{x}): \varepsilon(\mathbf{x})\rangle-\langle\mathbf{E}(\mathbf{x}) \cdot \mathcal{E}(\mathbf{x}): \varepsilon(\mathbf{x})\rangle \\
& -\frac{1}{2}\langle\mathbf{E}(\mathbf{x}) \cdot \boldsymbol{\alpha}(\mathbf{x}) \cdot \mathbf{E}(\mathbf{x})\rangle,
\end{aligned}
$$

introducing (38) in (39) in (42) and comparing the different terms of the resulting equation with (24), we obtain, after some calculations:

$$
\begin{aligned}
& \overline{\mathbb{C}}=\left\langle\left(\mathbb{A}^{0}(\mathbf{x})\right)^{T}: \mathbb{C}(\mathbf{x}): \mathbb{A}^{0}(\mathbf{x})\right. \\
& \left.-2\left(\mathcal{D}^{0}(\mathbf{x})\right)^{T} \cdot \mathcal{E}(\mathbf{x}): \mathbb{A}^{0}(\mathbf{x})-\left(\mathcal{D}^{0}(\mathbf{x})\right)^{T} \cdot \boldsymbol{\alpha}(\mathbf{x}) \cdot \mathcal{D}^{0}(\mathbf{x})\right\rangle
\end{aligned}
$$

$$
\begin{aligned}
& \overline{\boldsymbol{\alpha}}=\left\langle-\left(\mathcal{B}^{0}(\mathbf{x})\right)^{T}: \mathbb{C}(\mathbf{x}): \mathcal{B}^{0}(\mathbf{x})\right. \\
& \left.+2\left(\mathbf{h}^{0}(\mathbf{x})\right)^{T} \cdot \mathcal{E}(\mathbf{x}): \mathcal{B}^{0}(\mathbf{x})+\left(\mathbf{h}^{0}(\mathbf{x})\right)^{T} \cdot \boldsymbol{\alpha}(\mathbf{x}) \cdot \mathbf{h}^{0}(\mathbf{x})\right\rangle
\end{aligned}
$$




$$
\begin{aligned}
& \overline{\mathbb{G}}=\left\langle\left(\tilde{\mathbb{A}}^{1}(\mathbf{x})\right)^{T}: \mathbb{C}(\mathbf{x}): \tilde{\mathbb{A}}^{1}(\mathbf{x})\right. \\
& \left.-2\left(\tilde{\mathcal{D}}^{1}(\mathbf{x})\right)^{T} \cdot \mathcal{E}(\mathbf{x}): \tilde{\mathbb{A}}^{1}(\mathbf{x})-\left(\tilde{\mathcal{D}}^{1}(\mathbf{x})\right)^{T} \cdot \boldsymbol{\alpha}(\mathbf{x}) \cdot \tilde{\mathcal{D}}^{1}(\mathbf{x})\right\rangle,
\end{aligned}
$$

$$
\begin{aligned}
& \overline{\mathcal{E}}=\left\langle-\left(\mathcal{B}^{0}(\mathbf{x})\right)^{T}: \mathbb{C}(\mathbf{x}): \mathbb{A}^{0}(\mathbf{x})+\left(\mathbf{h}^{0}(\mathbf{x})\right)^{T} \cdot \mathcal{E}(\mathbf{x}): \mathbb{A}^{0}(\mathbf{x})\right. \\
& \left.+\left(\mathcal{B}^{0}(\mathbf{x})\right)^{T}: \mathcal{E}(\mathbf{x}) \cdot \mathcal{D}^{0}(\mathbf{x})+\left(\mathbf{h}^{0}(\mathbf{x})\right)^{T} \cdot \boldsymbol{\alpha}(\mathbf{x}) \cdot \mathcal{D}^{0}(\mathbf{x})\right\rangle
\end{aligned}
$$

$$
\begin{aligned}
& \overline{\mathbb{F}}=\left\langle\left(\mathcal{B}^{0}(\mathbf{x})\right)^{T}: \mathbb{C}(\mathbf{x}): \tilde{\mathbb{A}}^{1}(\mathbf{x})-\left(\mathbf{h}^{0}(\mathbf{x})\right)^{T} \cdot \mathcal{E}(\mathbf{x}): \tilde{\mathbb{A}}^{1}(\mathbf{x})\right. \\
& \left.-\left(\mathcal{B}^{0}(\mathbf{x})\right)^{T}: \mathcal{E}^{T}(\mathbf{x}) \cdot \tilde{\mathcal{D}}^{1}(\mathbf{x})-\left(\mathbf{h}^{0}(\mathbf{x})\right)^{T} \cdot \boldsymbol{\alpha}(\mathbf{x}) \cdot \tilde{\mathcal{D}}^{1}(\mathbf{x})\right\rangle
\end{aligned}
$$

$$
\begin{aligned}
& \overline{\mathbb{M}}=\left\langle\left(\mathbb{A}^{0}(\mathbf{x})\right)^{T}: \mathbb{C}(\mathbf{x}): \tilde{\mathbb{A}}^{1}(\mathbf{x})-\left(\mathcal{D}^{0}(\mathbf{x})\right)^{T} \cdot \mathcal{E}(\mathbf{x}): \tilde{\mathbb{A}}^{1}(\mathbf{x})\right. \\
& \left.-\left(\mathbb{A}^{0}(\mathbf{x})\right)^{T}: \mathcal{E}^{T}(\mathbf{x}) \cdot \tilde{\mathcal{D}}^{1}(\mathbf{x})-\left(\mathbb{D}^{0}(\mathbf{x})\right)^{T} \cdot \boldsymbol{\alpha}(\mathbf{x}) \cdot \tilde{\mathcal{D}}^{1}(\mathbf{x})\right\rangle
\end{aligned}
$$

\subsection{Vector and matrix forms of effective tensors}

In this work, we only consider composites with infinitely long parallel fibers. Then, we consider $2 \mathrm{D}$ plane strain conditions. In this case, the $2 \mathrm{D}$ vector and matrix forms of the different tensors are provided in what follows. The vector 
form associated with the nonsymmetric components of $\overline{\nabla \varepsilon}$ can be written as:

$$
[\overline{\nabla \varepsilon}]=\left[\begin{array}{l}
(\overline{\nabla \varepsilon})_{111} \\
(\overline{\nabla \varepsilon})_{221} \\
2(\overline{\nabla \varepsilon})_{122} \\
(\overline{\nabla \varepsilon})_{222} \\
(\overline{\nabla \varepsilon})_{112} \\
2(\overline{\nabla \varepsilon})_{121}
\end{array}\right]=\left[\begin{array}{l}
\frac{\partial^{2} u_{1}}{\partial x_{1}^{2}} \\
\frac{\partial^{2} u_{2}}{\partial x_{1} \partial x_{2}} \\
\frac{\partial^{2} u_{1}}{\partial x_{2}^{2}}+\frac{\partial^{2} u_{2}}{\partial x_{1} \partial x_{2}} \\
\frac{\partial^{2} u_{2}}{\partial x_{2}^{2}} \\
\frac{\partial^{2} u_{1}}{\partial x_{1} \partial x_{2}} \\
\frac{\partial^{2} u_{1}}{\partial x_{1} \partial x_{2}}+\frac{\partial^{2} u_{2}}{\partial x_{1}^{2}}
\end{array}\right]
$$

We then introduce the vector containing the components of the hyperstress tensor $\overline{\mathcal{S}}$ as:

$$
[\overline{\mathcal{S}}]=\left[\begin{array}{l}
\overline{\mathcal{S}}_{111} \\
\overline{\mathcal{S}}_{221} \\
\overline{\mathcal{S}}_{122} \\
\overline{\mathcal{S}}_{222} \\
\overline{\mathcal{S}}_{112} \\
\overline{\mathcal{S}}_{121}
\end{array}\right]
$$

In matrix form, the constitutive equations (26), (27), and (28) are given by:

$$
\begin{aligned}
& {[\overline{\mathbf{d}}]=[\mathcal{E}]^{T}[\overline{\boldsymbol{\varepsilon}}]+[\overline{\boldsymbol{\alpha}}] \overline{\mathbf{E}}-[\overline{\mathbb{F}}][\overline{\nabla \varepsilon}],} \\
& {[\overline{\boldsymbol{\sigma}}]=[\overline{\mathbb{C}}][\bar{\varepsilon}]-[\overline{\mathcal{E}}] \overline{\mathbf{E}}+[\overline{\mathbf{M}}][\overline{\nabla \varepsilon}],} \\
& {[\overline{\mathcal{S}}]=[\overline{\mathbf{M}}]^{T}[\overline{\boldsymbol{\varepsilon}}]+[\overline{\mathbb{F}}]^{T} \overline{\mathbf{E}}+[\overline{\mathbb{G}}][\overline{\nabla \boldsymbol{\varepsilon}}],}
\end{aligned}
$$

where $[\overline{\mathbf{d}}]$ is a column vector, and

$$
\begin{aligned}
{[\overline{\boldsymbol{\alpha}}] } & =\left[\begin{array}{ll}
\bar{\alpha}_{11} & \bar{\alpha}_{12} \\
\bar{\alpha}_{12} & \bar{\alpha}_{22}
\end{array}\right], \\
{[\overline{\mathbb{C}}] } & =\left[\begin{array}{lll}
\bar{C}_{1111} & \bar{C}_{1122} & \bar{C}_{1112} \\
\bar{C}_{1122} & \bar{C}_{2222} & \bar{C}_{2212} \\
\bar{C}_{1112} & \bar{C}_{2212} & \bar{C}_{1212}
\end{array}\right],
\end{aligned}
$$




$$
\begin{aligned}
& {[\overline{\mathcal{E}}]=\left[\begin{array}{ll}
\overline{\mathcal{E}}_{111} & \overline{\mathcal{E}}_{211} \\
\overline{\mathcal{E}}_{122} & \overline{\mathcal{E}}_{222} \\
\overline{\mathcal{E}}_{112} & \overline{\mathcal{E}}_{212}
\end{array}\right],} \\
& {[\overline{\mathbb{F}}]=\left[\begin{array}{llllll}
\bar{F}_{1111} & \bar{F}_{1221} & \bar{F}_{1122} & \bar{F}_{1222} & \bar{F}_{1112} & \bar{F}_{1121} \\
\bar{F}_{2111} & \bar{F}_{2221} & \bar{F}_{2122} & \bar{F}_{2222} & \bar{F}_{2112} & \bar{F}_{2121}
\end{array}\right],}
\end{aligned}
$$

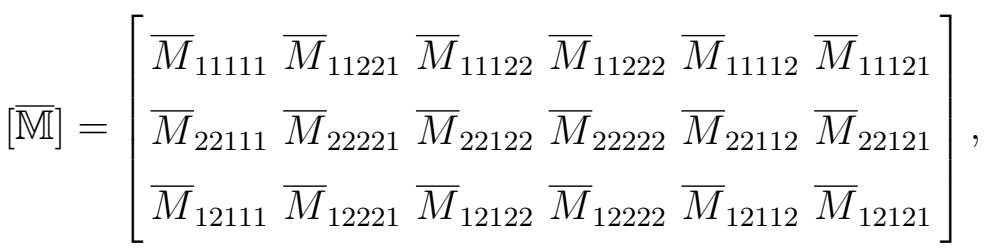

and

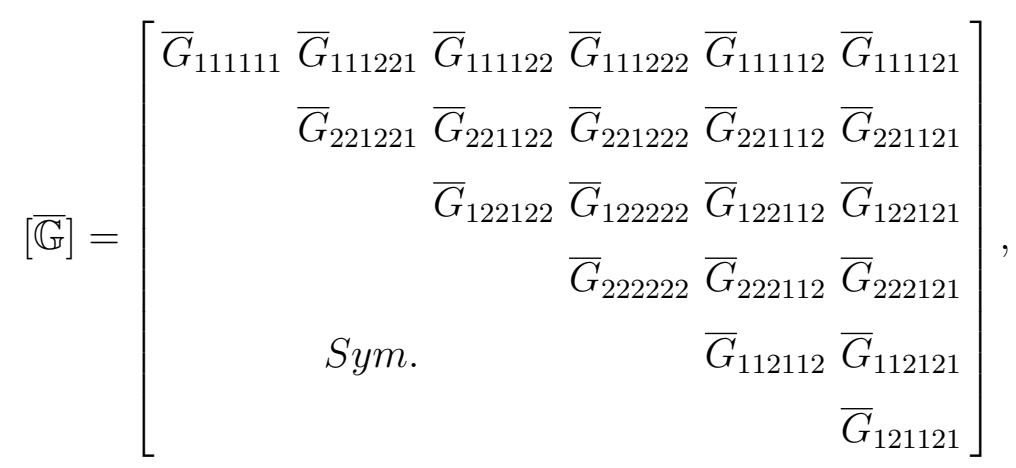

where we have taken into account the symmetries of these tensors, which are expressed as:

$$
\begin{aligned}
& \overline{\mathcal{E}}_{i j k}=\overline{\mathcal{E}}_{i k j}, \quad \overline{\mathbb{F}}_{i j k l}=\overline{\mathbb{F}}_{i k j l}, \\
& \overline{\mathbb{C}}_{i j k l}=\overline{\mathbb{C}}_{k l i j}=\overline{\mathbb{C}}_{j i k l}=\overline{\mathbb{C}}_{i j l k}, \\
& \overline{\mathbb{G}}_{i j k l m p}=\overline{\mathbb{G}}_{l m p i j k}=\overline{\mathbb{G}}_{j i k l m p}=\overline{\mathbb{G}}_{i j k m l p}, \\
& \overline{\mathbb{M}}_{i j k l m}=\overline{\mathbb{M}}_{j i k l m}=\overline{\mathbb{M}}_{i j l k m} .
\end{aligned}
$$




\section{Numerical investigation}

\subsection{Composite with piezoelectric phases}

In this first example, we investigate the effective flexoelectric properties of a two-phase periodic composite whose phase are both made of piezoelectric materials. The representative Volume Elements (RVE) are composed of periodic unit cells. Two geometries are investigated: one with triangular inclusions (Fig. 2(a)) and one with a fully asymmetric geometry as depicted in Fig. 2(b). Both geometries are chosen to limit the number of symmetries to increase the gradient effects. We assume that both phase are made with the same piezoelectric material but that there is a rotation mismatch between the main directions of the crystal in phases 1 and 2 by an angle $\theta$, which creates a material heterogeneity as the different mechanical, dielectric and piezoelectric tensors are assumed to be fully anisotropic.

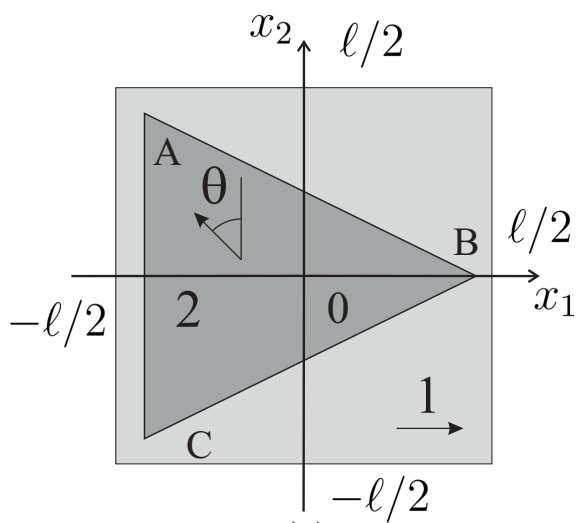

(a)

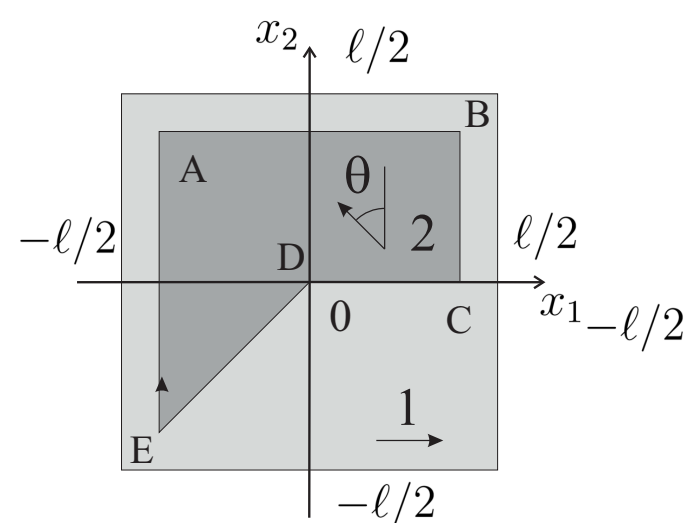

(b)

Fig. 2. (a) unit cell with triangular inclusions; (b) asymmetric unit cell.

The geometric description of the different unit cells is provided below. For the unit cell with triangular inclusions (Fig. 2 (a)), $A=\{-a \ell ; a \ell\}, B=$ $\{a \ell ; 0\}, C=\{-a \ell ;-a \ell\}$. For the unit with asymmetric inclusions (Fig. $2(\mathrm{~b})), A=\{-b \ell ; b \ell\}, B=\{b \ell ; b \ell\}, C=\{b \ell ; 0\}, D=\{0 ; 0\}, E=$ $\{-b \ell ;-b \ell\}$., with $a=\sqrt{0.8 \ell} / 2$ and $b=0.4 \ell$. The parameter $a$ is chosen such that both unit cells correspond to the same volume fraction $f=0.4$. Then, the RVE is assumed to be made of $N \times N$ unit cells. Unless otherwise specified, the length of the RVE is chosen as $L=N \ell$, with $\ell=1 \mathrm{~mm}$.

The inclusions (material 2 in Fig. 2) are made of a transversely anisotropic ceramic (lead zirconium titanate - PZT) whose parameters are given in 2D, and for an orientation of the piezoelectric crystal main direction along $x_{1}$, by 
[68]:

$$
\begin{aligned}
& {\left[\mathbf{C}^{1}\right]=\left[\begin{array}{ccc}
131.39 & 83.237 & 0 \\
83.237 & 154.837 & 0 \\
0 & 0 & 35.8
\end{array}\right](G P a)} \\
& {\left[\mathcal{E}^{1}\right]=\left[\begin{array}{ccc}
-2.120582 & -2.120582 & 0 \\
0 & 0 & 0
\end{array}\right]\left({\left.\mathrm{C} . \mathrm{m}^{-2}\right)}^{-2}\right.} \\
& {\left[\boldsymbol{\alpha}^{1}\right]=\left[\begin{array}{ll}
2.079 & 0 \\
0 & 4.065
\end{array}\right]\left(\mathrm{nC} \cdot \mathrm{m}^{-1} \cdot \mathrm{V}^{-1}\right)}
\end{aligned}
$$

Then, the properties of phase 2 (inclusion) are defined with respect to the angle $\theta$ according to:

$$
\begin{aligned}
& \alpha_{i j}^{2}=R_{i p} R_{j q} \alpha_{p q}^{1}, \\
& \mathcal{E}_{i j k}^{2}=R_{i p} R_{j q} R_{k r} \mathcal{E}_{p q r}^{1}, \\
& \mathbb{C}_{i j k l}^{2}=R_{i p} R_{j q} R_{k r} R_{l s} \mathbb{C}_{p q r s}^{1},
\end{aligned}
$$

where $\mathbf{R}$ is a rotation matrix defined by:

$$
\mathbf{R}=\left[\begin{array}{l}
\cos (\theta)-\sin (\theta) \\
\sin (\theta) \cos (\theta)
\end{array}\right]
$$

Examples of deformed configurations corresponding to the elementary loads are depicted in Fig. 3.

We first investigate the convergence of the effective flexoelectric properties with respect to the number of unit cells $N$ along each direction in the RVE. The triangular unit cell (Fig. 2 (a)) is chosen here. Results for the components $\overline{\mathbb{F}}_{1221}$ and $\overline{\mathbb{F}}_{2112}$ are provided in Fig. 4 . In this case, we have chosen $\theta=\pi$. We can appreciate a quick convergence with respect to $N$.

Next, we compute the evolution of some components of $\overline{\mathbb{F}}$ with respect to the mismatch angle $\theta$ for both triangular and asymmetric unit cells. We only depict the coefficients $\overline{\mathbb{F}}_{1221}, \overline{\mathbb{F}}_{2221}, \overline{\mathbb{F}}_{1112}$ and $\overline{\mathbb{F}}_{2112}$. These coefficients represent the polarization induced by bending, while the other coefficients are associated with polarization induced by more complex strain gradient modes, difficult to obtain practically. 


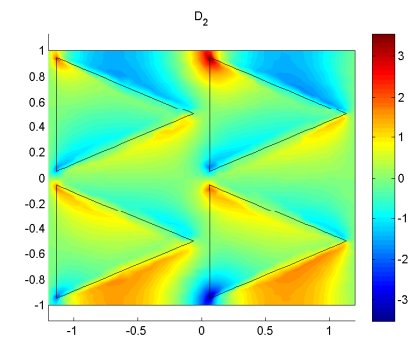

(a)

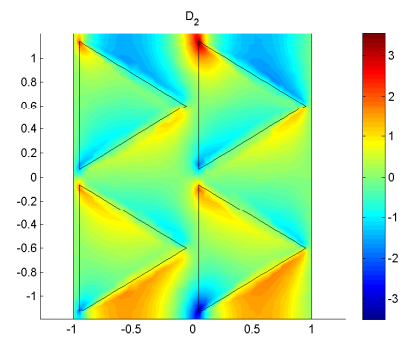

(b)

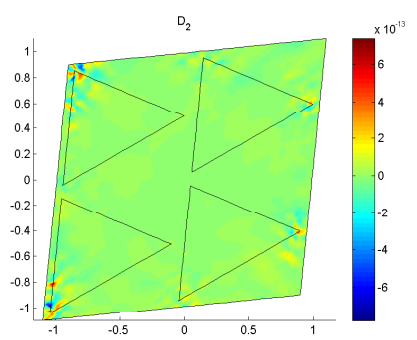

(c)

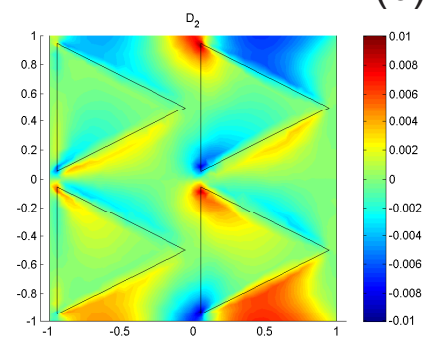

(d)

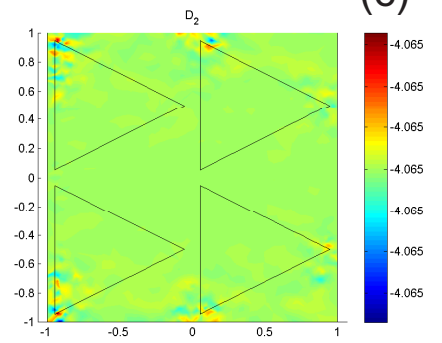

(e)

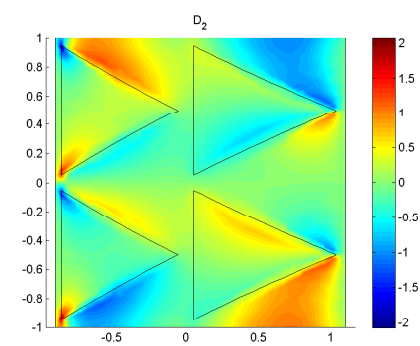

(f)

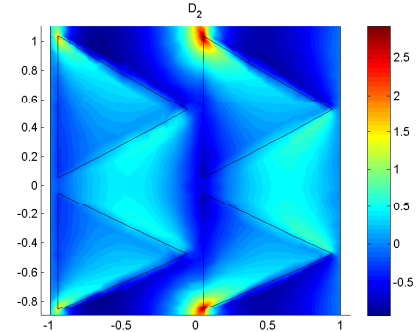

(i)

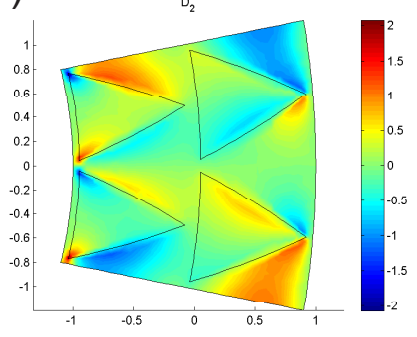

(g)

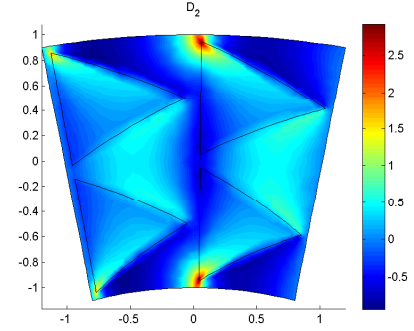

(j)

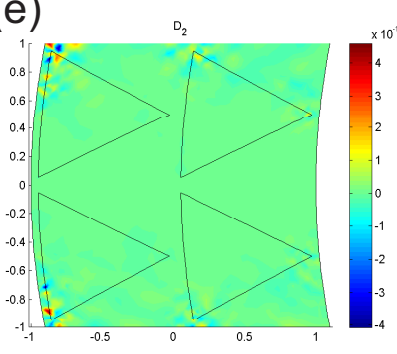

(h)

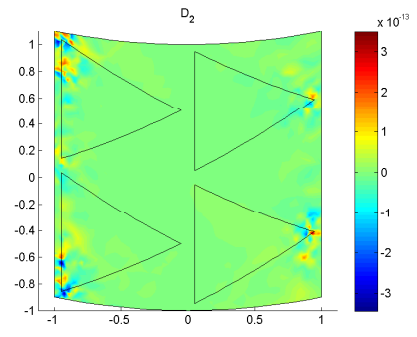

$(\mathrm{k})$

Fig. 3. $d_{2}(\mathbf{x})$ electric displacement field in deformed $(\times 0.2)$ configurations for RVE with triangular inclusions: (a) $[\bar{\varepsilon}]=[1 ; 0 ; 0], \overline{\nabla \varepsilon}=\mathbf{0}, \overline{\mathbf{E}}=\mathbf{0}$; (b) $[\bar{\varepsilon}]=[0 ; 1 ; 0]$, $\overline{\nabla \varepsilon}=\mathbf{0}, \overline{\mathbf{E}}=\mathbf{0} ;$ (c) $[\bar{\varepsilon}]=[0 ; 0 ; 1 / 2], \overline{\nabla \varepsilon}=\mathbf{0}, \overline{\mathbf{E}}=\mathbf{0} ;$ (d) $[\bar{\varepsilon}]=\mathbf{0}, \overline{\nabla \varepsilon}=\mathbf{0}$, $[\overline{\mathbf{E}}]=[1 ; 0] ;(\mathrm{e})[\overline{\boldsymbol{\varepsilon}}]=\mathbf{0}, \overline{\nabla \varepsilon}=\mathbf{0},[\overline{\mathbf{E}}]=[0 ; 1] ;$ (f) $[\bar{\varepsilon}]=\mathbf{0}, \overline{\nabla \varepsilon}=[1 ; 0 ; 0 ; 0 ; 0 ; 0]$, $\overline{\mathbf{E}}=\mathbf{0} ;(\mathrm{g})[\bar{\varepsilon}]=\mathbf{0}, \overline{\nabla \varepsilon}=[0 ; 1 ; 0 ; 0 ; 0 ; 0], \overline{\mathbf{E}}=\mathbf{0} ;(\mathrm{h})[\bar{\varepsilon}]=\mathbf{0}, \overline{\nabla \varepsilon}=[0 ; 0 ; 1 ; 0 ; 0 ; 0]$, $\overline{\mathbf{E}}=\mathbf{0}$; (i) $[\bar{\varepsilon}]=\mathbf{0}, \overline{\nabla \varepsilon}=[0 ; 0 ; 0 ; 1 ; 0 ; 0], \overline{\mathbf{E}}=\mathbf{0} ;$ (j) $[\overline{\boldsymbol{\varepsilon}}]=\mathbf{0}, \overline{\nabla \varepsilon}=[0 ; 0 ; 0 ; 0 ; 1 ; 0]$, $\overline{\mathbf{E}}=\mathbf{0} ;(\mathrm{k})[\overline{\boldsymbol{\varepsilon}}]=\mathbf{0}, \overline{\nabla \varepsilon}=[0 ; 0 ; 0 ; 0 ; 0 ; 1], \overline{\mathbf{E}}=\mathbf{0}$.

From Fig. 5 (triangular unit cell), we can first note that when $\theta=0$ (homogeneous medium), the flexoelectric effects vanish as expected. A maximum of the components $\overline{\mathbb{F}}_{1221}$ and $\overline{\mathbb{F}}_{2112}$ is found in this case for $\theta=\pi$, where both crystal phases are in the same direction but coefficients have opposite signs. The other components $\left(\overline{\mathbb{F}}_{2221}\right.$ and $\left.\overline{\mathbb{F}}_{1112}\right)$ have very small values as compared 


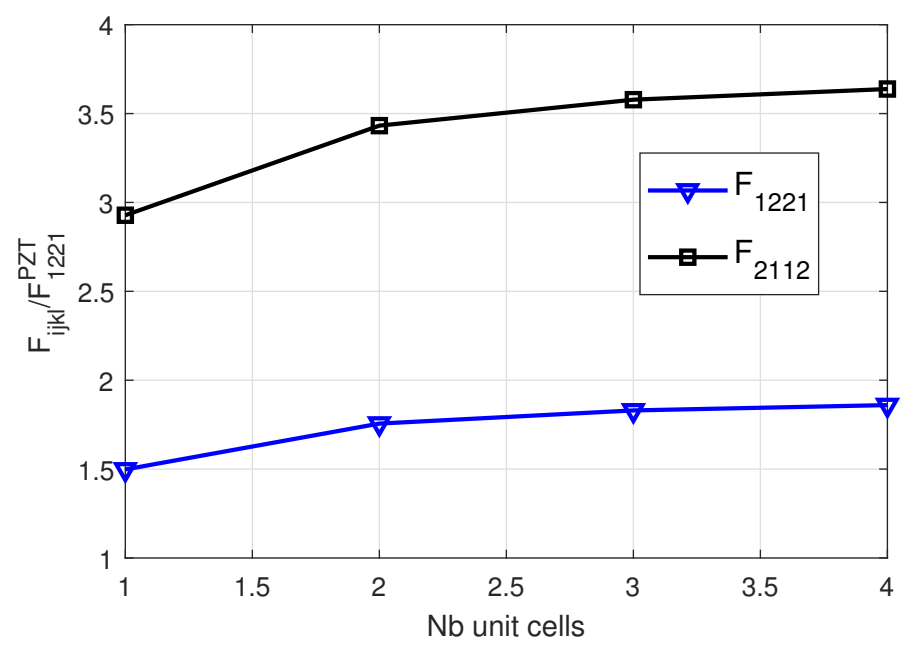

Fig. 4. Convergence of effective flexoelectric properties with the number of unit cells in the RVE.

to $\overline{\mathbb{F}}_{1221}$ and $\overline{\mathbb{F}}_{2112}$ and exhibit local minima.

In Fig. 6 (assymmetric unit cell), we can note that in this case the components $\overline{\mathbb{F}}_{1221}$ and $\overline{\mathbb{F}}_{2112}$ have two extrema with a maximum value for $\overline{\mathbb{F}}_{1221}$ at approximatively $\theta \simeq 1.2 \pi$.

Comparing both case, we can see that the maximum value of the coefficient $\overline{\mathbb{F}}_{2112}$, which corresponds to the variation of polarization in the direction $x_{2}$ with respect to bending around an out of plane axis $x_{3}$ (Fig. $\left.3(\mathrm{j})\right)$ is larger

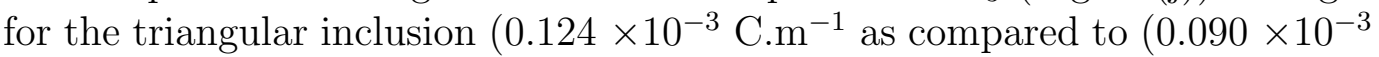
C. $\mathrm{m}^{-1}$ for the assymmetric case). Then we show that choosing appropriately direction mismatch between crystal directions and shapes, important increase of flexoelectric effects can be achieved. It is also worth noting that the obtained values are quite high as compared to naturally flexoelectric materials such as $\mathrm{BaTiO}_{3}$ whose flexoelectric coefficients are of the order of $10^{-5}$ C.m ${ }^{-1}$.

\subsection{Piezoelectric-elastic composite}

Next, a composite whose matrix is made of a piezoelectric material and an elastic inclusion is considered. The same geometries than in the previous example are considered (triangular shape, Fig. 2 (a)) and asymmetric geometry, Fig. 2 (b)). The material parameters of the matrix are the same as in the previous example (Eqs. (64), (65), (66)). The properties of the inclusions are 


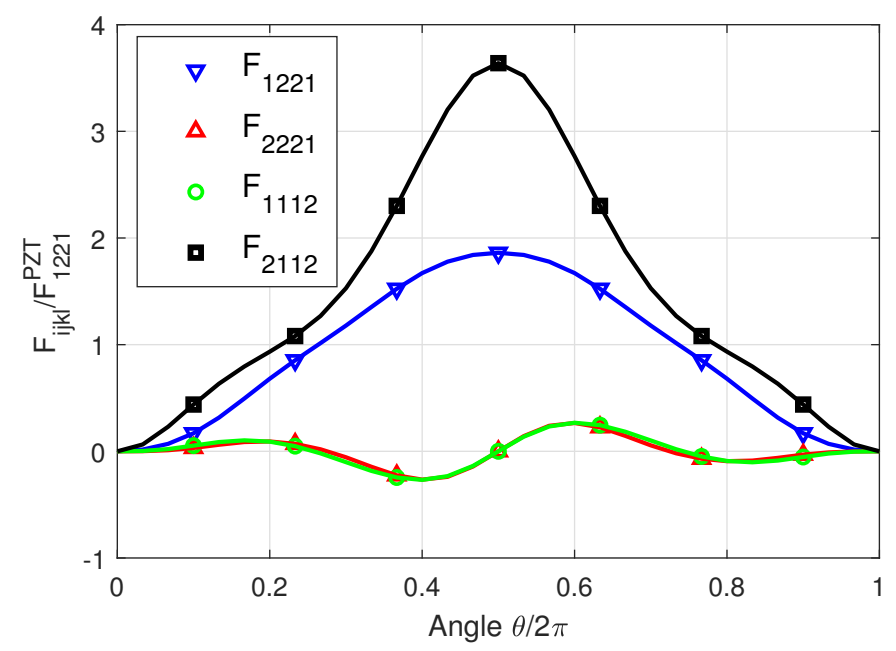

Fig. 5. Evolution of effective flexoelectric components with respect to the mismatch angle $\theta$ for the RVE with triangular inclusions.

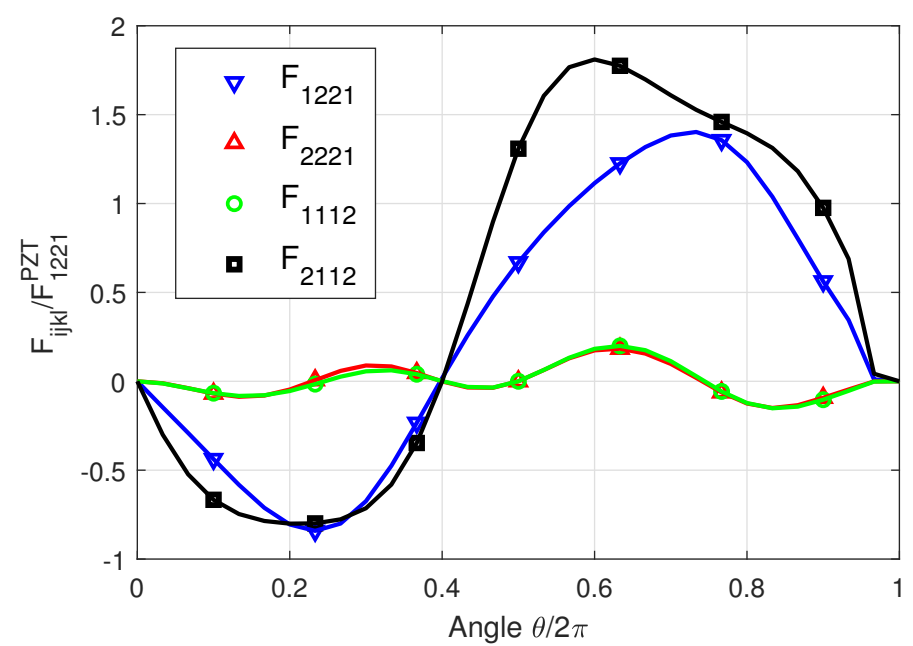

Fig. 6. Evolution of effective flexoelectric components with respect to the mismatch angle $\theta$ for the RVE with asymmetric inclusions.

here assumed to be linear isotropic elastic:

$$
\begin{aligned}
& {\left[\mathbf{C}^{2}\right]=\left[\begin{array}{llr}
\lambda^{i}+2 \mu^{i} & \lambda^{i} & 0 \\
\lambda^{i} & \lambda^{i}+2 \mu^{i} & 0 \\
0 & 0 & \mu^{i}
\end{array}\right](G P a)} \\
& {\left[\mathcal{E}^{2}\right]=\left[\begin{array}{lll}
0 & 0 & 0 \\
0 & 0 & 0
\end{array}\right]\left(\mathrm{C} \cdot \mathrm{m}^{-2}\right)}
\end{aligned}
$$




$$
\left[\boldsymbol{\alpha}^{2}\right]=\left[\begin{array}{cc}
\alpha^{i} & 0 \\
0 & \alpha^{i}
\end{array}\right]\left(\mathrm{nC} \cdot \mathrm{m}^{-1} \cdot \mathrm{V}^{-1}\right)
$$

with $\lambda^{i}$ and $\mu^{i}$ the Lamé's parameters related to the Young's modulus and Poisson's ratio through $\lambda^{i}=E^{i} \nu^{i} /\left(\left(1+\nu^{i}\right)\left(1-2 \nu^{i}\right)\right), \mu^{i}=E^{i} /\left(2\left(1+\nu^{i}\right)\right)$, and $\alpha^{i}$ is an isotropic dielectric coefficient. We first investigate the effect of varying the elastic modulus of the inclusion for a fixed Poisson's ratio $\nu^{i}=0.4$ and $\alpha^{i}=3.72 \times 10^{-2} \mathrm{nC} . \mathrm{m}^{-1} \cdot \mathrm{V}^{-1}$. Computations are conducted using $4 \times 4$ cells. Results are presented in Figs. 7 and 8 for the triangular and asymmetric shape, respectively. Surprisingly, the evolution of the effective coefficients is not monotonous and exhibit local extrema.

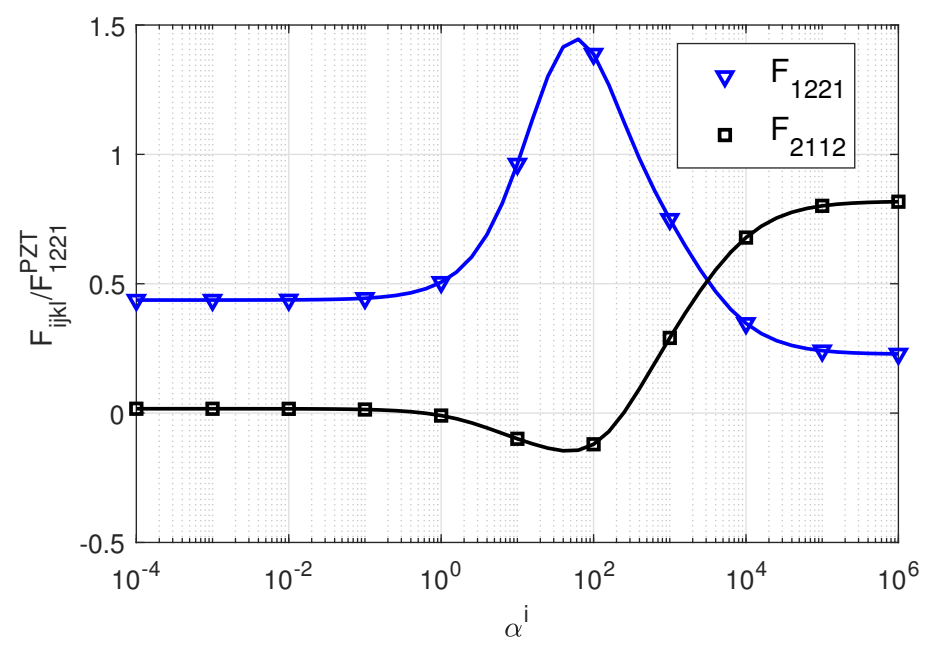

Fig. 7. Evolution of effective flexoelectric components with respect to the Young modulus of the inclusion for the RVE with triangular inclusions.

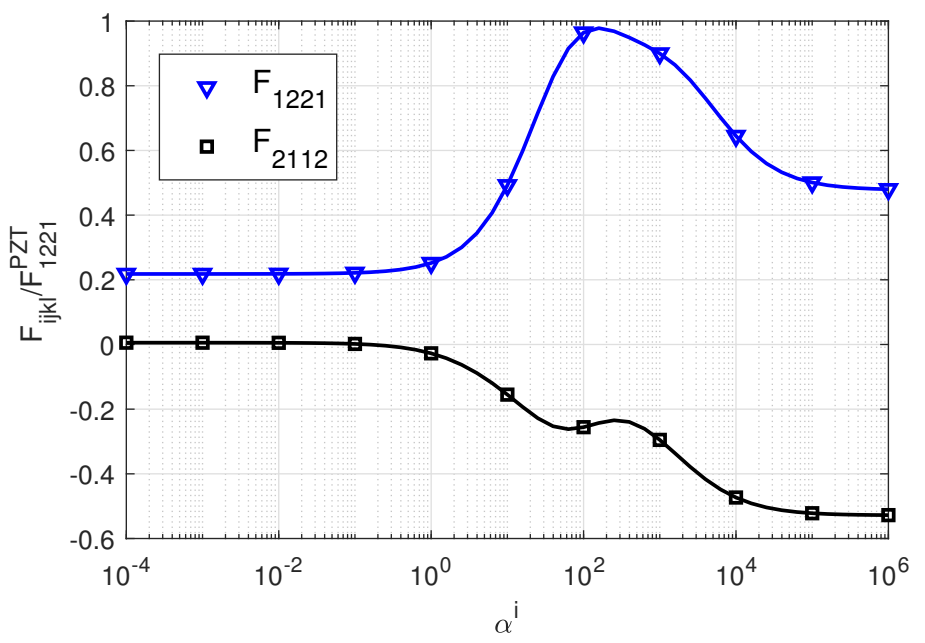

Fig. 8. Evolution of effective flexoelectric components with respect to the Young modulus of the inclusion for the RVE with asymmetric inclusions. 
Finally, we now evaluate the effect of varying the dielectric coefficient $\alpha^{i}$ for a fixed value $E^{i}=10^{2} \mathrm{GPa}, \nu^{i}=0.4$. Results are depicted in Figs. 9 and 10, exhibiting again non monotonous evolution of the flexoelectric coefficients with respect to the dielectric properties of the inclusion. Such complex evolutions show that such computational homogenization method could be used in future studies as a tool to optimize the effective flexoelectric properties of composite with respect to geometry and properties of phases.

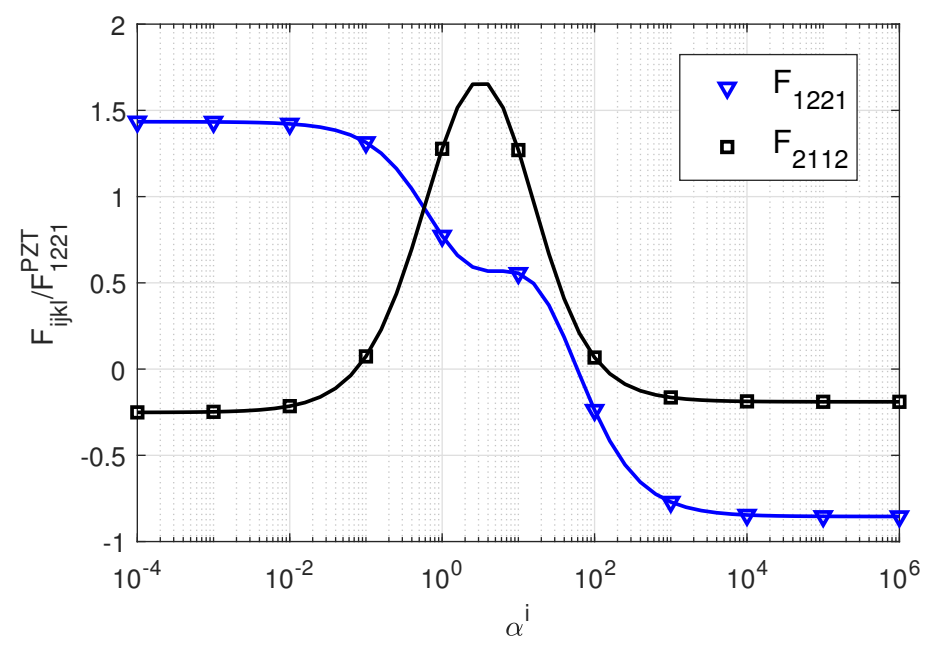

Fig. 9. Evolution of effective flexoelectric components with respect to dielectric properties the inclusion for the RVE with triangular inclusions.

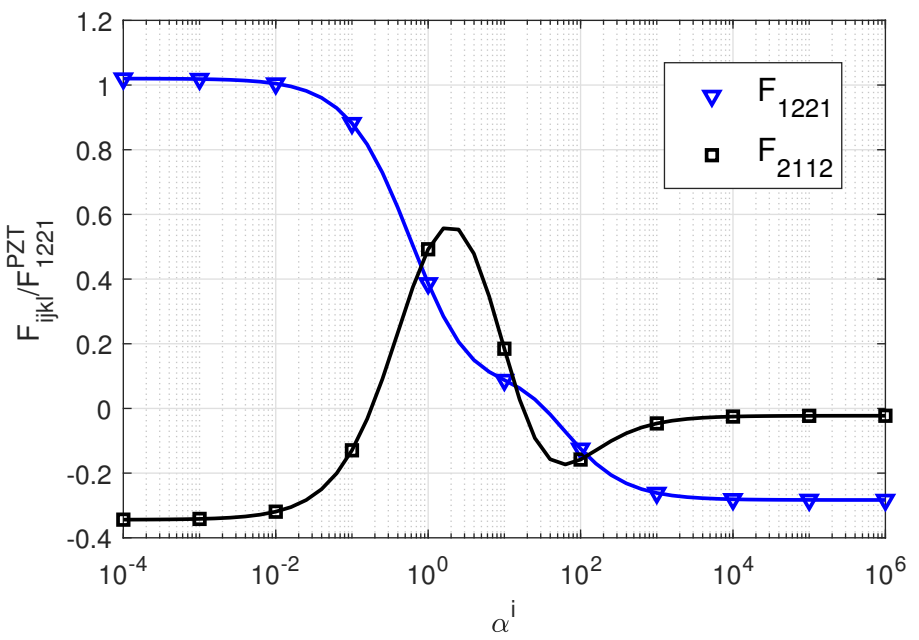

Fig. 10. Evolution of effective flexoelectric components with respect to dielectric properties of the inclusion for the RVE with asymmetric inclusions. 


\section{Concluding Remarks}

There are several instances of physical significance where we may prefer a flexoelectric response as opposed to piezoelectricity. In this work, we put concrete ideas on how to obtain a large effective flexoelectric response by creating composites constructed from piezoelectric phases. The constituents may exhibit negligible flexoelectricity. Specifically we have proposed a computational framework for estimating the effective linear flexoeletric behavior of such piezoelectric composites. As opposed to prior work, the present method allows evaluation of the full anisotropic flexoelectric tensor, by means of finite element numerical calculations over Representative Volume Elements (RVE), and then for arbitrary local geometries of phases. A corrected scheme has been proposed to remove spurious effects of previous strain gradient numerical homogenization schemes. Numerical investigations have been conducted to evaluate the evolution of flexoelectric coefficients with respect to local material properties and geometry of the composite phases, showing the potential of the approach for future optimization-based design of efficient flexoelectric systems and concomitant applications in fields such as energy harvesting.

\section{References}

[1] Gerda Buchberger, Reinhard Schwödiauer, and Siegfried Bauer. Flexible large area ferroelectret sensors for location sensitive touchpads. Applied Physics Letters, 92(12):123511, 2008.

[2] Federico Carpi, Danilo De Rossi, Roy Kornbluh, Ronald Edward Pelrine, and Peter Sommer-Larsen. Dielectric elastomers as electromechanical transducers: Fundamentals, materials, devices, models and applications of an emerging electroactive polymer technology. Elsevier, 2011.

[3] Dian Yang, Mohit S Verma, Ju-Hee So, Bobak Mosadegh, Christoph Keplinger, Benjamin Lee, Fatemeh Khashai, Elton Lossner, Zhigang Suo, and George M Whitesides. Buckling pneumatic linear actuators inspired by muscle. Advanced Materials Technologies, 1(3), 2016.

[4] Canan Dagdeviren, Byung Duk Yang, Yewang Su, Phat L Tran, Pauline Joe, Eric Anderson, Jing Xia, Vijay Doraiswamy, Behrooz Dehdashti, Xue Feng, et al. Conformal piezoelectric energy harvesting and storage from motions of the heart, lung, and diaphragm. Proceedings of the National Academy of Sciences, 111(5):1927-1932, 2014.

[5] Siegfried Bauer, Simona Bauer-Gogonea, Ingrid Graz, Martin Kaltenbrunner, Christoph Keplinger, and Reinhard Schwödiauer. 25th anniversary article: a soft future: from robots and sensor skin to energy harvesters. Advanced Materials, 26(1):149-162, 2014.

[6] Jiangshui Huang, Samuel Shian, Zhigang Suo, and David R Clarke. Max- 
imizing the energy density of dielectric elastomer generators using equibiaxial loading. Advanced Functional Materials, 23(40):5056-5061, 2013.

[7] Shengyou Yang, Xuanhe Zhao, and Pradeep Sharma. Avoiding the pullin instability of a dielectric elastomer film and the potential for increased actuation and energy harvesting. Soft Matter, 13:4552-4558, 2017.

[8] Federico Carpi, Siegfried Bauer, and Danilo De Rossi. Stretching dielectric elastomer performance. Science, 330(6012):1759-1761, 2010.

[9] John A Rogers, Takao Someya, and Yonggang Huang. Materials and mechanics for stretchable electronics. Science, 327(5973):1603-1607, 2010.

[10] P Muralt, RG Polcawich, and S Trolier-McKinstry. Piezoelectric thin films for sensors, actuators, and energy harvesting. MRS bulletin, 34(9):658-664, 2009.

[11] Alper Erturk and Daniel J Inman. Piezoelectric energy harvesting. John Wiley \& Sons, 2011.

[12] Heung Soo Kim, Joo-Hyong Kim, and Jaehwan Kim. A review of piezoelectric energy harvesting based on vibration. International journal of precision engineering and manufacturing, 12(6):1129-1141, 2011.

[13] Jun Ueda, Thomas W Secord, and H Harry Asada. Large effective-strain piezoelectric actuators using nested cellular architecture with exponential strain amplification mechanisms. IEEE/ASME transactions on mechatronics, 15(5):770-782, 2009.

[14] Jeong-Beom Ihn and Fu-Kuo Chang. Detection and monitoring of hidden fatigue crack growth using a built-in piezoelectric sensor/actuator network: I. diagnostics. Smart materials and structures, 13(3):609, 2004.

[15] Kenji Uchino. Piezoelectric actuators 2006. Journal of Electroceramics, 20(3-4):301-311, 2008.

[16] Lucia Seminara, Marco Capurro, Paolo Cirillo, Giorgio Cannata, and Maurizio Valle. Electromechanical characterization of piezoelectric pvdf polymer films for tactile sensors in robotics applications. Sensors and Actuators A: Physical, 169(1):49-58, 2011.

[17] L.P. Liu. An energy formulation of continuum magneto-electro-elasticity with applications. Journal of the Mechanics and Physics of Solids, 63:451-480, 2014.

[18] Seung-Bok Choi and Gi-Woo Kim. Measurement of flexoelectric response in polyvinylidene fluoride films for piezoelectric vibration energy harvesters. Journal of Physics D: Applied Physics, 50(7):075502, 2017.

[19] Wenhui Ma and L Eric Cross. Flexoelectricity of barium titanate. Applied Physics Letters, 88(23):232902, 2006.

[20] J.Y. Fu anf W. Zhu, N. Li, and L.E. Cross. Experimental studies of the converse flexoelectric effect induced by inhomogeneous electric field in a barium strontium titanate composition. Journal of Applied Physics, 100(2):024112, 2006.

[21] Eugene A Eliseev, Anna N Morozovska, Maya D Glinchuk, and R Blinc. Spontaneous flexoelectric/flexomagnetic effect in nanoferroics. Physical Review B, 79(16):165433, 2009. 
[22] Alexander G Petrov. Electricity and mechanics of biomembrane systems: flexoelectricity in living membranes. Analytica chimica acta, 568(1):7083, 2006.

[23] AT Todorov, AG Petrov, and JH Fendler. First observation of the converse flexoelectric effect in bilayer lipid membranes. The Journal of Physical Chemistry, 98(12):3076-3079, 1994.

[24] Fatemeh Ahmadpoor and Pradeep Sharma. Flexoelectricity in two-dimensional crystalline and biological membranes. Nanoscale, 7(40):16555-16570, 2015.

[25] Sana Krichen and Pradeep Sharma. Flexoelectricity: A perspective on an unusual electromechanical coupling. Journal of Applied Mechanics, 83(3):030801, 2016.

[26] P.V. Yudin and A.K. Tagantsev. Fundamentals of flexoelextricity in solids. Nanotechnology, 24:432001, 2013.

[27] P. Zubko, G. Catalan, and A.K. Tagantsev. Flexoelectric effect in solids. Annual Review of Materials Research, 43, 2013.

[28] Daesu Lee and Tae Won Noh. Giant flexoelectric effect through interfacial strain relaxation. Philosophical Transactions of the Royal Society of London A: Mathematical, Physical and Engineering Sciences, 370(1977):4944-4957, 2012.

[29] R Mbarki, N Baccam, Kaushik Dayal, and P Sharma. Piezoelectricity above the curie temperature? combining flexoelectricity and functional grading to enable high-temperature electromechanical coupling. Applied Physics Letters, 104(12):122904, 2014.

[30] Sheng Mao and Prashant K Purohit. Insights into flexoelectric solids from strain-gradient elasticity. Journal of Applied Mechanics, 81(8), 2014.

[31] MS Majdoub, P Sharma, and T Cagin. Enhanced size-dependent piezoelectricity and elasticity in nanostructures due to the flexoelectric effect. Physical Review B, 77(12):125424, 2008.

[32] Thanh D Nguyen, Sheng Mao, Yao-Wen Yeh, Prashant K Purohit, and Michael C McAlpine. Nanoscale flexoelectricity. Advanced Materials, 25(7):946-974, 2013.

[33] Qian Deng, Mejdi Kammoun, Alper Erturk, and Pradeep Sharma. Nanoscale flexoelectric energy harvesting. International Journal of Solids and Structures, 51(18):3218-3225, 2014.

[34] Qian Deng, Liping Liu, and Pradeep Sharma. Flexoelectricity in soft materials and biological membranes. Journal of the Mechanics and Physics of Solids, 62:209-227, 2014.

[35] A.H. Rahmati, S. Bauer, and P. Sharma. Nonlinear bending deformation of soft electrets and prospects for engineering flexoelectricity and transverse (d 31) piezoelectricity. Soft matter, 15(1):127-148, 2019.

[36] Xin Wen, Dongfan Li, Kai Tan, Qian Deng, and Shengping Shen. Flexoelectret: an electret with a tunable flexoelectriclike response. Physical review letters, 122(14):148001, 2019.

[37] Swapnil Chandratre and Pradeep Sharma. Coaxing graphene to be piezo- 
electric. Applied Physics Letters, 100(2):023114, 2012.

[38] Binglei Wang and Pradeep Sharma. Flexoelectricity as a universal mechanism for energy harvesting from crumpling of thin sheets. Physical Review B, 100(3):035438, 2019.

[39] Amir Abdollahi, Fabián Vásquez-Sancho, and Gustau Catalan. Piezoelectric mimicry of flexoelectricity. Physical review letters, 121(20):205502, 2018.

[40] E.R. Cholleti. A review on 3D printing of piezoelectric materials. In IOP Conference Series: Materials Science and Engineering, volume 455, page 012046. IOP Publishing, 2018.

[41] D. Guinovart-Sanjuán, J. Merodio, J.C. López-Realpozo, K. Vajravelu, R. Rodríguez-Ramos, R. Guinovart-Díaz, J. Bravo-Castillero, and F.J. Sabina. Asymptotic homogenization applied to flexoelectric rods. Materials, 12(2):232, 2019.

[42] S. Sidhardh and M.C. Ray. Effective properties of flexoelectric fiberreinforced nanocomposite. Materials Today Communications, 17:114-123, 2018.

[43] V.A. Eremeyev, J.-F. Ganghoffer, V. Konopińska-Zmysłowska, and N.K. Uglov. Flexoelectricity and apparent piezoelectricity of a pantographic micro-bar. International Journal of Engineering Science, 149:103213, 2020.

[44] P. Mohammadi, L.P. Liu, and P. Sharma. A theory of flexoelectric membranes and effective properties of heterogeneous membranes. Journal of Applied Mechanics, 81(1), 2014.

[45] H.T. Chen, S.D. Zhang, A.K. Soh, and W.Y. Yin. Phase field modeling of flexoelectricity in solid dielectrics. Journal of Applied Physics, 118(3):034106, 2015.

[46] S.S. Nanthakumar, X. Zhuang, H.S. Park, and T. Rabczuk. Topology optimization of flexoelectric structures. Journal of the Mechanics and Physics of Solids, 105:217-234, 2017.

[47] H. Ghasemi, H.S. Park, and T. Rabczuk. A multi-material level set-based topology optimization of flexoelectric composites. Computer Methods in Applied Mechanics and Engineering, 332:47-62, 2018.

[48] Graeme Walter Milton. Extending the theory of composites to other areas of science, 2017.

[49] S. Mao, P.K. Purohit, and N. Aravas. Mixed finite-element formulations in piezoelectricity and flexoelectricity. Proceedings of the Royal Society A: Mathematical, Physical and Engineering Sciences, 472(2190):20150879, 2016.

[50] K. M. Hamdia, H. Ghasemi, X. Zhuang, N. Alajlan, and T. Rabczuk. Sensitivity and uncertainty analysis for flexoelectric nanostructures. Computer Methods in Applied Mechanics and Engineering, 337:95-109, 2018.

[51] A. Abdollahi, P.C Christian, M.D. Daniel, M. Arroyo, and A.I. Irene. Computational evaluation of the flexoelectric effect in dielectric solids. Journal of Applied Physics, 116(9):093502, 2014. 
[52] Jason Marshall and Kaushik Dayal. Atomistic-to-continuum multiscale modeling with long-range electrostatic interactions in ionic solids. Journal of the Mechanics and Physics of Solids, 62:137-162, 2014.

[53] Lun Yang and Kaushik Dayal. A completely iterative method for the infinite domain electrostatic problem with nonlinear dielectric media. Journal of Computational Physics, 230(21):7821-7829, 2011.

[54] M.-L. Feng and C.-C. Wu. A study of three-dimensional four-step braided piezo-ceramic composites by the homogenization method. Composites Sience and Technology, 61(13):1889-1898, 2001.

[55] R. Brenner. Numerical computation of the response of piezoelectric composites using fourier transform. Physical Review B, 79(18):184106, 2009.

[56] R.D. Mindlin. Micro-structure in linear elasticity. Archive for Rational Mechanics and Analysis, 16(1):51-78, 1964.

[57] R.D. Mindlin and N.N. Eshel. On first strain-gradient theories in linear elasticity. International Journal of Solids and Structures, 4(1):109-124, 1968.

[58] J. Yvonnet, N. Auffray, and V. Monchiet. Computational second-order homogenization of materials with effective anisotropic strain gradient behavior. International Journal of Solids and Structures, 191-192:434-448, 2020.

[59] M. Gologanu, J.-B. Leblond, G. Perrin, and J. Devaux. Recent extensions of gurson's model for porous ductile metals. In Continuum micromechanics, pages 61-130. Springer, 1997.

[60] S. Forest. Mechanics of generalized continua: construction by homogenizaton. Le Journal de Physique IV, 8(PR4):Pr4-39, 1998.

[61] S. Forest, F. Pradel, and K. Sab. Asymptotic analysis of heterogeneous cosserat media. International Journal of Solids and Structures, 38(2627):4585-4608, 2001.

[62] V. Monchiet, N. Auffray, and J. Yvonnet. Strain-gradient homogenization: a bridge between asymptotic expansion and quadratic boundary condition methods. Mechanics of Materials, 143:103309, 2020.

[63] J. Yvonnet. Computational Homogenization of Heterogeneous Materials with Finite Elements. Springer Nature, 2019.

[64] Shuling Hu and Shengping Shen. Electric field gradient theory with surface effect for nano-dielectrics. Computers, Materials \& Continua (CMC), 13(1):63, 2009.

[65] V.G. Kouznetsova, M.G.D. Geers, and W.A.M. Brekelmans. Multi-scale constitutive modeling of heterogeneous materials with gradient enhanced computational homogenization scheme. International Journal for $\mathrm{Nu}$ merical Methods in Engineering, 54:1235-1260, 2002.

[66] L. Kaczmarczyk, C.J. Pearce, and N. Bićanić. Scale transition and enforcement of rve boundary conditions in second-order computational homogenization. International Journal for Numerical Methods in Engineering, 74(3):506-522, 2008.

[67] J. Yvonnet and L.P. Liu. A numerical framework for modeling flexoelec- 
tricity and maxwell stress in soft dielectrics at finite strains. Computer Methods in Applied Mechanics and Engineering, 313:450-482, 2017.

[68] E.Z. Pettermann and S. Suresh. A comprehensive unit cell model: a study of coupled effects in piezoelectric 1-3 composites. International Journal of Solids and Structures, 37(39):5447-5464, 2000.

\section{Appendix: Numerical evaluation of effective tensors}

The discrete form associated with (38) and (39) is given by:

$$
\begin{aligned}
& {[\varepsilon(\mathbf{x})]=\mathbf{A}^{0}(\mathbf{x})[\bar{\varepsilon}]+\mathbf{B}^{0}(\mathbf{x}) \overline{\mathbf{E}}+\tilde{\mathbf{A}}^{1}(\mathbf{x})[\overline{\nabla \varepsilon}]} \\
& \mathbf{E}(\mathbf{x})=\mathbf{D}^{0}(\mathbf{x})[\bar{\varepsilon}]+\mathbf{h}^{0}(\mathbf{x}) \overline{\mathbf{E}}+\tilde{\mathbf{D}}^{1}[\overline{\nabla \varepsilon}]
\end{aligned}
$$

with

$$
\begin{aligned}
& \tilde{\mathbf{A}}^{1}(\mathbf{x})=\mathbf{A}^{1}(\mathbf{x})-\mathbf{A}_{x}^{0}(\mathbf{x}) \\
& \tilde{\mathbf{D}}^{1}(\mathbf{x})=\mathbf{D}^{1}(\mathbf{x})-\mathbf{D}_{x}^{0}(\mathbf{x}) .
\end{aligned}
$$

Before defining the different above matrices, we introduce the matrices:

$$
\mathbf{U}_{u}=\left[\mathbf{u}^{1} ; \mathbf{u}^{2} ; \mathbf{u}^{3}\right] ; \mathbf{V}_{u}=\left[\mathbf{u}^{4} ; \mathbf{u}^{5}\right] ; \mathbf{W}_{u}=\left[\mathbf{u}^{6} ; \mathbf{u}^{7} ; \mathbf{u}^{8} ; \mathbf{u}^{9} ; \mathbf{u}^{10} ; \mathbf{u}^{11}\right]
$$

where $\mathbf{u}^{1}, \mathbf{u}^{2}$, and $\mathbf{u}^{3}$ are the respective vector columns containing the nodal displacement solution of the problem (18)-(23) with $\bar{\varepsilon}_{11}=1$, all other strain components to zero, $\overline{\mathbf{E}}=\mathbf{0}, \overline{\nabla \boldsymbol{\varepsilon}}=\mathbf{0}, \bar{\varepsilon}_{22}=1$, all other strain components to zero, $\overline{\mathbf{E}}=\mathbf{0}, \overline{\nabla \boldsymbol{\varepsilon}}=\mathbf{0}, \bar{\varepsilon}_{12}=1 / 2$, all other strain components to zero, $\overline{\mathbf{E}}=\mathbf{0}, \overline{\nabla \varepsilon}=\mathbf{0}, \mathbf{u}^{4}, \mathbf{u}^{5}$ are respectively the vector columns containing the nodal displacement solution of the problem (18)-(23) with $\bar{E}_{1}=1$, all other electric field components to zero, $\bar{\varepsilon}=\mathbf{0}, \overline{\nabla \varepsilon}=\mathbf{0}, \bar{E}_{2}=1$, all other electric field components to zero, $\overline{\boldsymbol{\varepsilon}}=\mathbf{0}, \overline{\nabla \boldsymbol{\varepsilon}}=\mathbf{0}$, and $\mathbf{u}^{6}, \mathbf{u}^{7}, \mathbf{u}^{8}, \mathbf{u}^{9}, \mathbf{u}^{10}, \mathbf{u}^{11}$ are the respective vector columns containing the nodal displacement solution of the problem (18)-(23) with $\overline{\nabla \varepsilon}_{111}=1$, all other components of the strain gradient tensor to zero, $\bar{\varepsilon}=\mathbf{0}, \overline{\mathbf{E}}=\mathbf{0}, \bar{\nabla}_{221}=1$, all other components of the strain gradient tensor to zero, $\overline{\boldsymbol{\varepsilon}}=\mathbf{0}, \overline{\mathbf{E}}=\mathbf{0}, \bar{\nabla}_{121}=1$, all other components of the strain gradient tensor to zero, $\overline{\boldsymbol{\varepsilon}}=\mathbf{0}, \overline{\mathbf{E}}=\mathbf{0}, \bar{\nabla}_{222}=1$, all other components of the strain gradient tensor to zero, $\overline{\boldsymbol{\varepsilon}}=\mathbf{0}, \overline{\mathbf{E}}=\mathbf{0}, \bar{\nabla}_{112}=1$, all other components of the strain gradient tensor to zero, $\overline{\boldsymbol{\varepsilon}}=\mathbf{0}, \overline{\mathbf{E}}=\mathbf{0}, \bar{\nabla}_{121}=1$, all other components of the strain gradient tensor to zero, $\overline{\boldsymbol{\varepsilon}}=\mathbf{0}, \overline{\mathbf{E}}=\mathbf{0}$. 
Furthermore, we define:

$$
\mathbf{U}_{\phi}=\left[\phi^{1} ; \phi^{2} ; \phi^{3}\right] ; \mathbf{V}_{\phi}=\left[\phi^{4} ; \phi^{5}\right] ; \mathbf{W}_{\phi}=\left[\phi^{6} ; \phi^{7} ; \phi^{8} ; \phi^{9} ; \phi^{10} ; \phi^{11}\right]
$$

where $\phi^{1}, \phi^{2}, \phi^{3}$ are the respective vector columns containing the nodal electric potentials solution of the problem (18)-(23) with $\bar{\varepsilon}_{11}=1$, all other strain components to zero, $\overline{\mathbf{E}}=\mathbf{0}, \overline{\nabla \varepsilon}=\mathbf{0}, \bar{\varepsilon}_{22}=1$, all other strain components to zero, $\overline{\mathbf{E}}=\mathbf{0}, \overline{\nabla \varepsilon}=\mathbf{0}, \bar{\varepsilon}_{12}=1 / 2$, all other strain components to zero, $\overline{\mathbf{E}}=\mathbf{0}$, $\overline{\nabla \varepsilon}=\mathbf{0}, \phi^{4}, \phi^{5}$ are respective the vector columns containing the nodal electric potentials solution of the problem (18)-(23) with $\bar{E}_{1}=1$, all other electric field components to zero, $\bar{\varepsilon}=\mathbf{0}, \overline{\nabla \varepsilon}=\mathbf{0}, \bar{E}_{2}=1$, all other electric field components to zero, $\bar{\varepsilon}=\mathbf{0}, \overline{\nabla \varepsilon}=\mathbf{0}$, and $\phi^{6}, \phi^{7}, \phi^{8}, \phi^{9}, \boldsymbol{\phi}^{10}, \mathbf{u}^{11}$ are the respective vector columns containing the nodal electric potentials solution of the problem (18)-(23) with $\nabla \varepsilon_{111}=1$, all other components of the strain gradient tensor to zero, $\overline{\boldsymbol{\varepsilon}}=\mathbf{0}, \overline{\mathbf{E}}=\mathbf{0}, \bar{\nabla}_{221}=1$, all other components of the strain gradient tensor to zero, $\overline{\boldsymbol{\varepsilon}}=\mathbf{0}, \overline{\mathbf{E}}=\mathbf{0}, \bar{\nabla}_{122}=1$, all other components of the strain gradient tensor to zero, $\overline{\boldsymbol{\varepsilon}}=\mathbf{0}, \overline{\mathbf{E}}=\mathbf{0}, \bar{\nabla}_{222}=1$, all other components of the strain gradient tensor to zero, $\overline{\boldsymbol{\varepsilon}}=\mathbf{0}, \overline{\mathbf{E}}=\mathbf{0}, \bar{\nabla}_{112}=1$, all other components of the strain gradient tensor to zero, $\overline{\boldsymbol{\varepsilon}}=\mathbf{0}, \overline{\mathbf{E}}=\mathbf{0}, \bar{\nabla}_{121}=1$, all other components of the strain gradient tensor to zero, $\bar{\varepsilon}=\mathbf{0}, \overline{\mathbf{E}}=\mathbf{0}$.

Using the above definitions, we set:

$$
\begin{aligned}
& \mathbf{A}^{0}(\mathbf{x})=\mathbf{B}(\mathbf{x}) \mathbf{U}_{u} \\
& \mathbf{B}^{0}(\mathbf{x})=\mathbf{B}(\mathbf{x}) \mathbf{V}_{u} \\
& \mathbf{A}^{1}(\mathbf{x})=\mathbf{B}(\mathbf{x}) \mathbf{W}_{u} \\
& \mathbf{A}_{x}^{0}(\mathbf{x})=\mathbf{B}(\mathbf{x}) \mathbf{W}_{u}^{x}
\end{aligned}
$$

with

$$
\mathbf{W}_{u}^{x}(\mathbf{x})=\left[x \times \mathbf{u}^{1} ; y \times \mathbf{u}^{1} ; x \times \mathbf{u}^{2} ; y \times \mathbf{u}^{2} ; x \times \mathbf{u}^{3} ; y \times \mathbf{u}^{3}\right]
$$

and

$$
\begin{aligned}
& \mathbf{D}^{0}(\mathbf{x})=-\mathbf{B}_{\phi}(\mathbf{x}) \mathbf{U}_{\phi} \\
& \mathbf{h}^{0}(\mathbf{x})=-\mathbf{B}_{\phi}(\mathbf{x}) \mathbf{V}_{\phi} \\
& \mathbf{D}^{1}(\mathbf{x})=-\mathbf{B}_{\phi}(\mathbf{x}) \mathbf{W}_{\phi} \\
& \mathbf{D}_{x}^{0}(\mathbf{x})=-\mathbf{B}_{\phi}(\mathbf{x}) \mathbf{W}_{\phi}^{x}
\end{aligned}
$$


with

$$
\mathbf{W}_{\phi}^{x}(\mathbf{x})=\left[x \times \phi^{1} ; y \times \phi^{1} ; x \times \phi^{2} ; y \times \phi^{2} ; x \times \phi^{3} ; y \times \phi^{3}\right]
$$

Introducing (74) and (75) in (4.2)-(48) we obtain:

$$
[\overline{\mathbb{C}}]=\left\langle\left(\mathbf{A}^{0}\right)^{T}[\mathbb{C}] \mathbf{A}^{0}(\mathbf{x})-2\left(\mathbf{D}^{0}\right)^{T}[\mathcal{E}] \mathbf{A}^{0}-\left(\mathbf{D}^{0}\right)^{T}[\boldsymbol{\alpha}] \mathbf{D}^{0}\right\rangle
$$

$$
[\overline{\boldsymbol{\alpha}}]=\left\langle-\left(\mathbf{B}^{0}\right)^{T}[\mathbb{C}] \mathbf{B}^{0}+2\left(\mathbf{h}^{0}\right)^{T}[\mathcal{E}] \mathbf{B}^{0}+\left(\mathbf{h}^{0}\right)^{T}[\boldsymbol{\alpha}]\left[\mathbf{h}^{0}\right]\right\rangle
$$

$$
[\overline{\mathbb{G}}]=\left\langle\left(\tilde{\mathbf{A}}^{1}\right)^{T} \mathbf{C} \tilde{\mathbf{A}}^{1}-2\left(\tilde{\mathbf{D}}^{1}\right)^{T}[\mathcal{E}] \tilde{\mathbf{A}}^{1}-\left(\tilde{\mathbf{D}}^{1}\right)^{T}[\boldsymbol{\alpha}] \tilde{\mathbf{D}}^{1}\right\rangle
$$

$$
[\overline{\mathcal{E}}]=\left\langle-\left(\mathbf{B}^{0}\right)^{T}[\mathbb{C}] \mathbf{A}^{0}+\left(\mathbf{h}^{0}\right)^{T}[\mathcal{E}] \mathbf{A}^{0}+\left(\mathbf{B}^{0}\right)^{T}[\mathcal{E}] \mathbf{D}^{0}+\left(\mathbf{h}^{0}\right)^{T}[\boldsymbol{\alpha}] \mathbf{D}^{0}\right\rangle
$$

$$
[\overline{\mathbb{F}}]=\left\langle\left(\mathbf{B}^{0}\right)^{T}[\mathbb{C}] \tilde{\mathbf{A}}^{1}-\left(\mathbf{h}^{0}\right)^{T}[\mathcal{E}] \tilde{\mathbf{A}}^{1}-\left(\mathbf{B}^{0}\right)^{T}[\mathcal{E}]^{T} \tilde{\mathbf{D}}^{1}-\left(\mathbf{h}^{0}\right)^{T}[\boldsymbol{\alpha}] \tilde{\mathbf{D}}^{1}\right\rangle
$$

$$
[\overline{\mathbf{M}}]=\left\langle\left(\mathbf{A}^{0}\right)^{T}[\mathbb{C}] \tilde{\mathbf{A}}^{1}-\left(\mathbf{D}^{0}\right)^{T}[\mathcal{E}] \tilde{\mathbf{A}}^{1}-\left(\mathbf{A}^{0}\right)^{T}[\mathcal{E}] \tilde{\mathbf{D}}^{1}-\left(\mathbf{D}^{0}\right)^{T}[\boldsymbol{\alpha}] \tilde{\mathbf{D}}^{1}\right\rangle
$$

where we have omitted the dependence to $\mathbf{x}$ to alleviate the notations. 


\section{Appendix: FEM discretization of local RVE equations}

In this section we introduce the weak form and the FEM discretization for piezoelectricity in 2D. The weak form associated with the coupled problem (18)-(19) is given by:

$$
\begin{gathered}
\int_{\Omega} \mathbf{D} \cdot \nabla(\delta \phi) d \Omega=-\int_{\Omega}\{\overline{\mathcal{E}}: \overline{\nabla \boldsymbol{\varepsilon}} \mathbf{x}\} \cdot \nabla(\delta \phi) d \Omega \\
\int_{\Omega} \boldsymbol{\sigma}: \boldsymbol{\varepsilon}(\delta \mathbf{u}) d \Omega=\int_{\Omega} \nabla \cdot\{\overline{\mathbb{C}}: \overline{\nabla \boldsymbol{\varepsilon}} \mathbf{x}\} \boldsymbol{\varepsilon}(\delta \mathbf{u}) d \Omega .
\end{gathered}
$$

Introducing (9) and (8) in (90)-(91) yields:

$$
\begin{aligned}
& \int_{\Omega}(\mathcal{E}: \boldsymbol{\varepsilon}(\mathbf{u})+\boldsymbol{\alpha} \cdot \mathbf{E}(\phi)) \cdot \nabla(\delta \phi) d \Omega=-\int_{\Omega}\{\overline{\mathcal{E}}: \overline{\nabla \varepsilon} \mathbf{x}\} \cdot \nabla(\delta \phi) d \Omega \\
& \int_{\Omega}\left(\mathbb{C}: \boldsymbol{\varepsilon}(\mathbf{u})-\mathcal{E}^{T} \cdot \mathbf{E}(\phi)\right): \boldsymbol{\varepsilon}(\delta \mathbf{u}) d \Omega=\int_{\Omega} \nabla \cdot\{\overline{\mathbb{C}}: \bar{\nabla} \boldsymbol{\varepsilon} \mathbf{x}\}: \varepsilon(\delta \mathbf{u}) d \Omega
\end{aligned}
$$

Introducing classical FEM discretization in (92)-(93) we obtain the linear system of coupled equations:

$$
\left[\begin{array}{cc}
\mathbf{K}_{\phi \phi} & \mathbf{K}_{\phi u} \\
-\mathbf{K}_{\phi u}^{T} & \mathbf{K}_{u u}
\end{array}\right]\left[\begin{array}{c}
\boldsymbol{\phi}^{e} \\
\mathbf{u}^{e}
\end{array}\right]=\left[\begin{array}{c}
\mathbf{F}_{\phi} \\
\mathbf{F}_{u}
\end{array}\right]
$$

with

$$
\begin{aligned}
\mathbf{K}_{\phi \phi} & =\int_{\Omega}\left(\mathbf{B}_{\phi}\right)^{T}[\boldsymbol{\alpha}] \mathbf{B}_{\phi} d \Omega, \\
\mathbf{K}_{\phi u} & =\int_{\Omega}\left(\mathbf{B}_{\phi}\right)^{T}[\mathcal{E}] \mathbf{B} d \Omega, \\
\mathbf{K}_{u u} & =\int_{\Omega}(\mathbf{B})^{T}[\mathbb{C}] \mathbf{B} d \Omega,
\end{aligned}
$$

where $\mathbf{B}_{\phi}$ with and $\mathbf{B}$ are shape function derivatives such that $\nabla(\phi)=\mathbf{B}_{\phi} \boldsymbol{\phi}^{e}$ and $[\varepsilon]=\mathbf{B} \mathbf{u}^{e}$, where $\boldsymbol{\phi}^{e}$ and $\mathbf{u}^{e}$ denote nodal potential and displacement vectors, respectively, and $[\varepsilon]$ denotes the vector form od the strain tensor $\varepsilon$.

$$
\begin{aligned}
\mathbf{F}_{\phi} & =-\int_{\Omega}\left(\mathbf{B}_{\phi}\right)^{T}[\mathcal{E}][\boldsymbol{\eta}] \\
\mathbf{F}_{u} & =\int_{\Omega} \mathbf{B}^{T}[\mathbb{C}][\boldsymbol{\eta}]
\end{aligned}
$$


and

$$
[\boldsymbol{\eta}]=\left[\begin{array}{l}
\bar{\nabla}_{111} x_{1}+\bar{\nabla}_{112} x_{2} \\
\bar{\nabla}_{221} x_{1}+\bar{\nabla}_{222} x_{2} \\
\bar{\nabla} \varepsilon_{121} x_{1}+\bar{\nabla}_{122} x_{2}
\end{array}\right] .
$$

